\title{
Auditory Plasticity in a Basal Ganglia-Forebrain Pathway during Decrystallization of Adult Birdsong
}

\author{
Arani Roy and Richard Mooney \\ Department of Neurobiology, Duke University Medical Center, Durham, North Carolina 27710
}

\begin{abstract}
Adult male zebra finches maintain highly stable songs via auditory feedback. Prolonged exposure to distorted feedback may cause this stable (i.e., "crystallized") song to change its pattern, a process known as decrystallization. In the songbird, the telencephalic nucleus LMAN (lateral magnocellular nucleus of anterior nidopallium) is necessary for feedback-dependent song decrystallization, although whether and how electrophysiological properties of LMAN neurons change during decrystallization is unknown. In normal adult zebra finches, LMAN neurons exhibit highly selective responses to auditory presentation of the bird's own song (BOS), possibly providing a permanent referent for song maintenance. If so, LMAN neurons should maintain selectivity for the originally crystallized BOS after exposure to distorted feedback and during decrystallization. Alternatively, LMAN auditory selectivity in the adult may change during decrystallization. To distinguish between these possibilities, we sectioned the vocal nerve in adult male zebra finches, which spectrally distorted the birds' songs. Over the course of several weeks, experience of distorted feedback caused the song to decrystallize in a subset of birds. At various times after nerve section, electrophysiological recordings made under anesthesia revealed that auditory selectivity in LMAN could shift to the spectrally distorted song. Such auditory plasticity could be detected during the second week after nerve section, before the time birds typically decrystallized their songs. Moreover, all birds that underwent decrystallization at later times always manifested auditory plasticity in LMAN. To our knowledge, the present findings afford the first example of an electrophysiological correlate of song decrystallization.
\end{abstract}

Key words: songbird; zebra finch; LMAN; auditory feedback; auditory selectivity; vocal plasticity; vocal nerve; basal ganglia

\section{Introduction}

Birdsong is a paradigmatic example of a critical period limited learned behavior (Immelmann, 1969; Marler, 1970; Doupe and Kuhl, 1999). Juvenile songbirds learn to sing by using auditory feedback to match their variable song patterns to a memorized tutor model (Konishi, 1965; Marler and Waser, 1977). After critical period closure, song crystallizes into a highly stereotyped pattern less susceptible to auditory feedback perturbations. However, in adult zebra finches, spectral and temporal features of a previously crystallized song will slowly change (i.e., decrystallize) when feedback is disrupted by deafening (Nordeen and Nordeen, 1992), superimposing altered feedback during singing (Leonardo and Konishi, 1999; Zevin et al., 2004), or transecting the vocal nerve (Williams and McKibben, 1992). In songbirds, the telencephalic nucleus LMAN (lateral magnocellular nucleus of anterior nidopallium) links a basal ganglia-forebrain pathway [anterior forebrain pathway (AFP)] to song premotor areas (see Fig. 1A) (Nottebohm et al., 1976). Notably, LMAN lesions prevent dis-

Received Sept. 11, 2006; revised April 27, 2007; accepted May 1, 2007.

This work was supported by National Institutes of Health Grant DC02524 (R.M.). We thank Drs. David Fitzpatrick, Stephen Shea, Timothy Gentner, and J. Martin Wild for useful comments on a preliminary draft of this manuscript and Dr. John Prather and Stephan Nenkov for help with Matlab coding. We thank the two anonymous reviewers and the senior editor at The Journal of Neuroscience for useful comments. The stimulating discussions with the various members of the Mooney laboratory have benefited this manuscript enormously, and we thank them all.

Correspondence should be addressed to Dr. Richard Mooney, Department of Neurobiology, Duke University Medical Center, Box 3209, Durham, NC 27710. E-mail: mooney@neuro.duke.edu.

DOI:10.1523/JNEUROSCI.0894-07.2007

Copyright $\odot 2007$ Society for Neuroscience $\quad$ 0270-6474/07/276374-14\$15.00/0 rupted auditory feedback from triggering song decrystallization (Williams and Mehta, 1999; Brainard and Doupe, 2000a). Although LMAN is necessary for adult vocal plasticity, how LMAN neuronal properties change during decrystallization is unknown.

LMAN neurons in adult songbirds respond strongly to auditory presentation of the bird's own song (BOS), but not to temporally altered versions of the BOS or songs of other conspecific birds (Doupe and Konishi, 1991; Doupe, 1997; Rosen and Mooney, 2000). This selectivity develops in parallel with song learning (Solis and Doupe, 1997) and is compromised by chronically disrupting auditory feedback during song learning (Solis and Doupe, 2000), suggesting that auditory feedback shapes LMAN selectivity (Brainard and Doupe, 2000b). However, whether selectivity in LMAN shifts during decrystallization is unknown. At one extreme, BOS selectivity may be permanently fixed after crystallization, perhaps serving as an auditory referent for song maintenance. Conversely, LMAN neurons may continue to shift their selectivity during decrystallization. A third possibility is that strong BOS-evoked responses in LMAN depend on the similarity of the BOS to the tutor model. In support of this idea, juvenile zebra finches subjected to vocal nerve section fail to accurately imitate the tutor song and subsequently, as adults, lack auditory responses in LMAN (Solis and Doupe, 2000). This matching model predicts that LMAN auditory responses in adults should diminish after manipulations that distort the adult BOS and trigger decrystallization.

To distinguish between these possibilities, we severed the vo- 
cal nerve in adult male zebra finches, which immediately distorted spectral features of the songs but in most birds triggered changes to song temporal patterns only $2-3$ weeks later (the delayed process defined here as decrystallization) (Williams and McKibben, 1992). In vivo intracellular and extracellular recordings from such birds under urethane anesthesia allowed measurements of LMAN auditory selectivity before or after distorted feedback triggered song decrystallization (see Fig. $1 B$ ). In some birds, selectivity could shift during the second week after nerve section, in the absence of decrystallization. Moreover, all birds that underwent decrystallization at later times contained LMAN neurons selective for decrystallized BOS. Thus, LMAN neurons remain plastic beyond the critical period, rather than affording a permanent referent for song maintenance or indicating the quality of the match between BOS and the intended target.

\section{Materials and Methods}

Animals and experimental groups. Experiments were performed on a total of 51 adult (age, $138 \pm 12 \mathrm{~d}$ posthatch; mean $\pm \mathrm{SD}$; range, $110-155 \mathrm{~d}$ ) male zebra finches (Taeniopygia guttata) in accordance with a protocol approved by the Duke University Institutional Animal Care and Use Committee. The birds were divided up in three main experimental groups: "first-week" birds $(n=12)$ that were electrophysiologically recorded between 2 and $7 \mathrm{~d}$ after vocal nerve section; "second-week" birds ( $n=17$; of these, two birds were discarded for electrophysiology because of song decrystallization) (see supplemental Fig. $5 C$, available at www. jneurosci.org as supplemental material) that were recorded between 8 and $17 \mathrm{~d}$ after vocal nerve section; and "long-term" birds $(n=22)$ that were recorded at $>21 \mathrm{~d}$ after vocal nerve section. After analysis of song temporal structure (see below), the long-term birds were further divided in to "plastic" $(n=8)$ and "non-plastic" $(n=14)$ groups. The mean interval between nerve section and electrophysiological recordings was comparable between plastic and non-plastic birds (plastic: $61.4 \pm 11.9$; range, $29-125 \mathrm{~d}$; non-plastic: $58.7 \pm 7.1$; range, $21-116 \mathrm{~d}$; error bars denote SEM).

For analysis of LMAN auditory properties in normal adults, LMAN intracellular data were obtained from a previously published study (Rosen and Mooney, 2000). In this larger data set, song recordings from 21 birds contained sufficient material for conducting song stereotypy measurements. The strength and selectivity of auditory responses were analyzed in a total of 63 LMAN neurons in these 21 birds in which intracellular recordings to at least 10 iterations of BOS playback were available. For analysis of response variability, an additional subset of LMAN neurons ( $n=20$ neurons in 13 birds) with at least 20 iterations of BOS playback was selected from this pool. For analysis of response variability in juveniles, intracellular recordings ( $n=26$ neurons) were obtained from LMAN neurons in a separate set of five juvenile zebra finches (age, $76 \pm 9 \mathrm{~d}$ posthatch; mean $\pm \mathrm{SD}$; range, 65-85d).

Vocal (tracheosyringeal) nerve section. Before nerve section, songs of all birds were recorded in the presence of an adult female bird. Birds were then anesthetized with Equithesin $(2 \mathrm{mg} / \mathrm{kg}$, i.m.; $0.85 \mathrm{~g}$ of chloral hydrate, $0.21 \mathrm{~g}$ of pentobarbitol, $0.42 \mathrm{~g}$ of $\mathrm{MgSO}_{4}, 2.2 \mathrm{ml}$ of $100 \%$ ethanol, and $8.6 \mathrm{ml}$ of propylene glycol, brought to a final $20 \mathrm{ml}$ volume with distilled $\mathrm{H}_{2} \mathrm{O}$ ), and a 3-5 mm section of the right tracheosyringeal nerve was removed, followed by a resuturing of the skin. Postmortem visual inspection in a random sample of birds showed that the nerve did not grow back during the following weeks' to months' time. After surgery, birds were allowed to recover alone in a solitary cage for $1 \mathrm{~d}$ and either transferred to the main colony where they shared cages with other birds or kept in isolation such that they could not see but could hear other birds. This difference in holding conditions did not, however, have any bearing on the behavioral or physiological outcomes of vocal nerve section. Reorganization of song temporal structure as a long-term consequence of nerve section and shifts in LMAN auditory selectivity were observed in both isolated and nonisolated birds. Therefore, for all of the experiments described in this study, data from the two groups were always pooled together.
Song recording and spectral similarity measurement. Songs were recorded before and at regular intervals $(\sim 7 \mathrm{~d})$ after vocal nerve section in the presence of an adult female zebra finch. All song analyses described here were performed on female-directed songs only. Song recording and editing were performed using custom software (LabView; National Instruments, Austin, TX). Song spectrograms were subsequently created using the software Sound Analysis-2 (Tchernichovski et al., 2000). For analyzing spectral and temporal changes in song after nerve section, first we randomly chose single song motifs (excluding introductory notes) from each song recording session in each bird. Then, to quantify degradation in spectral quality of song after nerve section, we used the "percentage significant similarity" measure in Sound Analysis-2. Briefly, this measurement contrasts acoustic features such as pitch, Wiener entropy, sound continuity, and frequency modulation (FM) between small segments of two given songs and returns a statistical estimate of overall similarity between the two songs in the form of percentage significant similarity score. We calculated percentage significant similarity in the "overall" mode with the following settings: threshold, 92\%; interval, 70 ms; sections, $20 \mathrm{~ms}$. Using this technique, we first compared with each other 10 pairs of song motifs recorded before nerve section in each bird. This gave us a "pre-nerve section" estimate of spectral variance already existing in normal songs. Subsequently, 10 song motifs recorded at different intervals after nerve section were compared with 10 pre-nerve section motifs to obtain an estimate of the degradation in spectral qualities induced by the nerve section. Because song recordings in each bird were done on slightly different days after nerve section, direct averaging of similarity scores for one particular time point across birds was not possible. Therefore, to obtain a mean similarity measurement across birds, we pooled together from all birds the similarity scores obtained for the first, the second, and the last song recordings (marked post1, post2, and post3, respectively, in Fig. $1 B, 2 A$, and supplemental Fig. 5B, available at www.jneurosci.org as supplemental material) after nerve section and averaged them. In the long-term group of birds $(n=22)$, the first, the second, and the last song recordings were made at $7 \pm 1.4$ (mean \pm SEM), $14 \pm 2.9$, and $62 \pm 10 \mathrm{~d}$, respectively, after nerve section. In the second-week birds $(n=17)$, the respective recordings were made at $2 \pm$ $0.2,7 \pm 0.4$ and $11 \pm 0.9 \mathrm{~d}$ after nerve section.

Analysis of song temporal structure. For analysis of song temporal structure, amplitude envelopes of song motifs were cross-correlated using custom Matlab software. This analysis was based on the principle that if two song motifs are very similar to each other in their overall temporal structure (i.e., syllable sequence), cross-correlation between the amplitude waveforms of the two should return a high cross-correlation coefficient. We half-wave rectified the signal, then filtered and smoothed the rectified amplitude envelopes using the "decimate" function in Matlab (with the original sampling rate of $22,050 \mathrm{~Hz}$ and $r=10$ ). Next, we performed cross-correlation between these filtered amplitude envelopes using the "xcorr" function. The correlation coefficient was then normalized in the following manner: for a given waveform, the amplitudes of all of the points were squared and summed, and the square root of the sum was taken as the power in the signal. Then, the cross-correlation coefficient was divided by the product of the power in the two waveforms being compared. The peak normalized cross-correlation coefficient thus obtained ranged between 0 (indicating no similarity between the waveforms) and 1 (indicating identical waveforms).

Assessment of song decrystallization. Using this method, we compared song motifs of a single bird from 2 different days after nerve section, and the mean peak normalized cross-correlation coefficient thus obtained was defined as the "temporal integrity (TI) score," signifying the relative degree to which the song structure was stable between those two days. We also estimated the variability in song temporal structure on a given day by calculating the mean normalized cross-correlation coefficient for 10 randomly chosen motifs within that day. This value was defined as the "stereotypy score," which was essentially equivalent to a "within-bird, within-day" TI score. The stereotypy scores were calculated both before and on different days after vocal nerve section. Two groups of birds were analyzed using this technique: the long-term birds $(n=22)$ and the second-week birds $(n=17)$. In all birds encompassing both groups, the mean stereotypy scores before vocal nerve section were high (mean ste- 
reotypy score: long term, $0.86 \pm 0.05$; second week, $0.88 \pm 0.04$; mean \pm $\mathrm{SD})$. For a given group of birds (long term or second week), $2 \mathrm{SD}$ below the mean pre-nerve section stereotypy score was operationally defined as the threshold for estimating TI of songs after nerve section: after nerve section, any TI score falling below this threshold was used as the criterion for detecting song decrystallization.

After nerve section, we calculated for each bird a mean TI score by cross-correlating the amplitude envelopes of 10 song motifs from the first song recording session after nerve section (post1) with those of 10 song motifs from subsequent recording sessions. Comparison between the first and second recording sessions (post1 vs post2) (see Fig. 2A,B) provided an estimate of TI at "early" times after nerve section. Likewise, comparisons between the first and the last song recordings (post1 vs post3) gave us an estimate of TI at "late" times after nerve section. We did not compare the post-nerve section song motifs directly to the pre-nerve section song motifs for two reasons. First, although amplitude waveforms of songs immediately after nerve section roughly resembled that of pre-nerve section songs, the distortions in spectral features caused by the manipulation induced some alterations in the amplitude waveforms and thus made the comparisons less faithful purely to the temporal structure of song. Second, very rarely syllables (often those involving a high-pitch click) were dropped immediately after nerve section, potentially signifying that those syllables were being generated solely through the right syrinx. Thus, comparisons of pre- and early post-nerve section motifs in those birds returned lower TI scores that did not reflect auditory feedback-mediated alterations in the song temporal structure. Therefore, we took the earliest recording after nerve section as the starting point from where to monitor integrity of song temporal structure. Note that the TI score was not calculated for the first-week birds because in these birds only $1 \mathrm{~d}$ of song recording was typically obtained after nerve section. However, visual comparison of spectrograms of songs from before nerve section and during the first week after nerve section indicated that there were no obvious changes to the song temporal structure during this time.

In vivo electrophysiology and data analysis. On the morning of the day of the electrophysiological recording, birds were given injections in the pectoral muscle with $20 \%$ urethane (75-105 $\mu$ l total; Sigma, St. Louis, $\mathrm{MO}$ ), administered in three $25-35 \mu \mathrm{l}$ doses at $30 \mathrm{~min}$ intervals. LMAN recording sites were stereotaxically located and marked on the birds' skull. After topical application of xylocaine (2\%), the scalp was retracted, and a small craniotomy $(<300 \mu \mathrm{m}$ wide $)$ was made over spots marked for LMAN. A stainless steel post was affixed to the rostral part of the bird's skull with dental acrylic, which was used to immobilize the bird's head in a sound-attenuating chamber (Industrial Acoustics, Bronx, NY) on an air table (TMC, Peabody, MA); body temperature was maintained via an electric blanket at $37^{\circ} \mathrm{C}$ (Harvard Apparatus, Holliston, MA). After immobilizing the bird's head, the dura overlying the recording site was slit open with a fine insect pin (Minuten; Carolina Biological Supply, Burlington, NC).

Intracellular recordings were performed by sharp electrodes (borosilicate glass, BF100-50-10; Sutter Instruments, Novato, CA) with a resistance of 100-250 M $\Omega$ when filled with $3 \mathrm{M} \mathrm{K}$-acetate and 5\% neurobiotin. A hydraulic microdrive (Soma Scientific, Irvine, CA) was used to lower electrodes into LMAN ( $\sim 1.8 \mathrm{~mm}$ depth). An AxoClamp 2B intracellular amplifier (Molecular Devices, Foster City, CA) was used in bridge mode to record intracellular potentials, which were low-pass filtered at $3 \mathrm{kHz}$, digitized at $10 \mathrm{kHz}$, and stored on a personal computer (PC) hard drive. After conclusion of recording, cells were filled with neurobiotin using positive current pulses $(+0.5$ to $+1 \mathrm{nA}, 500 \mathrm{~ms}$ at 1 $\mathrm{Hz}$ ). LMAN neurons were identified on-line by their firing properties (Rosen and Mooney, 2000) and verified histologically after the recording session. Neurons were not selected based on any auditory response criterion, and all stably impaled neurons were recorded from. Peristimulus time histograms (PSTHs; $25 \mathrm{~ms}$ bin width) and median-filtered averaged membrane potential traces were computed on-line (see below, Data analysis).

Extracellular recordings in LMAN were performed using carbon-fiber microelectrodes (Carbostar-4, 0.4-0.8 M $\Omega$; Kation Scientific, Minneapolis, MN). Recorded extracellular signals were amplified via an A-M
Systems (Everett, WA) model 1700 differential amplifier, bandpass filtered between $300 \mathrm{~Hz}$ and $5 \mathrm{kHz}$, digitized at $10 \mathrm{kHz}$, and stored on a PC hard drive. LMAN sites were identified by their characteristic spontaneous bursting patterns, and all sites exhibiting such patterns were sampled from regardless of their auditory response properties. After conclusion of recordings, $5 \%$ biocytin was iontophoretically injected ( $2 \mu \mathrm{A}$ positive current, $7 \mathrm{~s}$ on-off cycle, for $8-10 \mathrm{~min}$ ) through the barrels of Carbostar-4 electrodes to mark the recording site.

Song stimuli. To record auditory responses, at least 15 iterations of each auditory stimulus, delivered at intervals ranging from 6 to $10 \mathrm{~s}$, were presented at $\sim 70 \mathrm{~dB}$ (rms, A-weighting) through a speaker positioned 20 $\mathrm{cm}$ directly in front of the bird. Two stimuli always presented during electrophysiological recordings included the BOS recorded before nerve section (old BOS) and post-nerve section song recorded just before electrophysiology (new BOS). The new BOS in the case of plastic birds represented a temporally reorganized song pattern, whereas in first-week, second-week, and non-plastic birds it represented a spectrally distorted but temporally intact song. The new and old BOS stimuli in all birds were chosen randomly, but in some plastic birds singing songs with variable durations, a new BOS motif was chosen that was comparable in duration to the old BOS. Further characterization of LMAN auditory selectivity was performed with various other auditory stimuli, including old and new versions of the BOS played in reverse (REVold and REVnew, respectively), songs of other adult zebra finches [conspecific (CON)], and songs from other nerve-sectioned zebra finches (tsCON). Additionally, in some plastic birds, an early version of the post-nerve section song that had not yet undergone decrystallization was used for playback (tsBOSold).

Data analysis. The suprathreshold responsiveness $\left(R_{\text {supra }}\right)$ of cells with spiking activity was calculated by $R_{\text {supra }}=S_{\mathrm{FR}}-B_{\mathrm{FR}}$, where $S_{\mathrm{FR}}$ and $B_{\mathrm{FR}}$ are the firing rates during each stimulus presentation and during a $1.5 \mathrm{~s}$ baseline period before each stimulus presentation, respectively. To assess subthreshold responsiveness in spiking and nonspiking cells, raw traces first were median filtered (each point was replaced by the median value of the surrounding 50 points, equivalent to $5 \mathrm{~ms}$ at the $10 \mathrm{kHz}$ sampling rate used here). Median filtering removed the action potential (which was typically $\sim 1 \mathrm{~ms}$ in duration), yet did not distort slower membrane potential movements. The subthreshold depolarizing responsiveness $\left(R_{\mathrm{Vm}}\right)$ of these cells was measured by $R_{\mathrm{Vm}}=S_{\text {area }}-B_{\text {area, }}$, where $S_{\text {area }}$ and $B_{\text {area }}$ are the integrals of the positive-going deviations in membrane potential either during (i.e., $S_{\text {area }}$ ) or before (i.e., $B_{\text {area }}$ ) stimulus presentation relative to the mode membrane potential measured during the baseline period. That is, the total positive area during the baseline (measured from the mode) was subtracted from the total positive area during the stimulus (measured from the mode). The mode membrane potential was calculated for the baseline data array with an automated LabView routine and was used instead of the mean or median of the baseline data array because we observed that it gave the most reliable measure of the central tendency of the baseline membrane potential. Average $R_{\text {supra }}$ or $R_{\mathrm{Vm}}$ was computed for at least 15 stimulus iterations. Significance was determined with paired $t$ tests comparing stimulus-evoked suprathreshold or subthreshold depolarizing responses to corresponding baseline measures. Subsequent response strength and selectivity measurements were performed only for neurons that exhibited significant auditory responses to at least one of the two versions of the BOS (i.e., the old or new BOS). Next, to compare intracellular and extracellular response strengths, and to compare suprathreshold and subthreshold responses, the response strengths calculated as above were converted to $Z$ scores. The suprathreshold $Z$ score $\left(Z_{\text {supra }}\right)$ is given by the difference between the average firing rate during stimulus presentation and that during a $1.5 \mathrm{~s}$ baseline period before stimulus presentation, divided by the SD of this difference:

$$
Z_{\text {supra }}=\frac{\bar{S}_{\mathrm{FR}}-\bar{B}_{\mathrm{FR}}}{\sqrt{\operatorname{Var}\left(S_{\mathrm{FR}}\right)+\operatorname{Var}\left(B_{\mathrm{FR}}\right)-2 \operatorname{Covar}\left(S_{\mathrm{FR}}, B_{\mathrm{FR}}\right)}},
$$

where $\overline{\mathrm{S}}_{\mathrm{FR}}$ is the mean firing rate during the stimulus, $\overline{\mathrm{B}}_{\mathrm{FR}}$ is the mean firing rate during the baseline period, and the denominator is the SD of $S_{\mathrm{FR}}-B_{\mathrm{FR}}$. For nonspiking cells and median-filtered spiking cells, the subthreshold $Z$ score $\left(Z_{\text {sub }}\right)$ is given by the difference between the average area during stimulus presentation and that during baseline, divided 
by the SD of this difference. The $Z_{\text {sub }}$ formula is the same as that for $Z_{\text {supra }}$ with substitutions of area $_{\text {for }}$, where $\bar{S}_{\text {area }}$ is the mean deviation in $V_{\mathrm{m}}$ (from the baseline mode, calculated separately for negative or positive area, as stated above) during song presentation and $\overline{\mathrm{B}}_{\text {area }}$ is the mean deviation in $V_{\mathrm{m}}$ during baseline; the denominator is the SD of $S_{\text {area }}-B_{\text {area }}$.

The selectivity of a given neuron for one auditory stimulus over another was measured using the psychophysical metric $d^{\prime}$, which estimates the discriminability between two stimuli. The $d^{\prime}$ value comparing the responses to new BOS relative to old BOS is given by the following:

$$
d^{\prime}{ }_{\text {N-O }) \text { supra }}=\frac{2\left(\bar{R}_{\mathrm{FRnew}}-\bar{R}_{\mathrm{FRold}}\right)}{\sqrt{\sigma_{\text {new }}^{2}+\sigma_{\text {old }}^{2}}},
$$

or

$$
d^{\prime}{ }_{\text {(N-O)sub }}=\frac{2\left(\bar{R}_{\text {AREAnew }}-\bar{R}_{\text {AREAold }}\right)}{\sqrt{\sigma_{\text {new }}^{2}+\sigma_{\text {old }}^{2}}},
$$

where $d^{\prime}{ }_{(\mathrm{N}-\mathrm{O}) \text { supra }}$ is suprathreshold selectivity and $d^{\prime}{ }_{(\mathrm{N}-\mathrm{O}) \text { sub }}$ is subthreshold selectivity. Similar calculations comparing other combinations of stimuli were also performed. $\overline{\mathrm{R}}$ is the mean value of $R$ (as described above), and $\sigma^{2}$ is its variance. Note that $d^{\prime}$ values for subthreshold responses $\left(d^{\prime}{ }_{\text {sub }}\right)$ can be calculated separately for positive and negative areas. Because previous studies have shown that BOS-evoked synaptic responses in LMAN are almost exclusively excitatory (Rosen and Mooney, 2000), for the purpose of this study, we reported values only for the positive area (i.e., for depolarizing subthreshold responses).

The single-unit and multiunit $d^{\prime}{ }_{\mathrm{N}-\mathrm{O}}$ values recorded in LMAN of a single bird were comparable (see supplemental Fig. $6 A, B$, available at www.jneurosci.org as supplemental material). This was confirmed by $t$ tests in birds with multiple single-unit and multiunit data points $(p>$ 0.05 in all cases). Therefore, we averaged all single-unit and multiunit data points recorded in each bird to calculate mean $d^{\prime}{ }_{\mathrm{N}-\mathrm{O}}$ values per bird. Unless stated otherwise, values of all electrophysiological parameters are reported as mean \pm SEM.

Measuring auditory response variability. To quantify the variability of auditory-evoked suprathreshold responses of LMAN neurons, we computed the mean spike time variability across multiple presentations of a song stimulus (for a schematic of the method, see supplemental Fig. 1, available at www.jneurosci.org as supplemental material). We first excerpted spike trains obtained during each stimulus presentation, maintaining the original alignment to the stimulus. Spike counts were then made at $10 \mathrm{~ms}$ bin resolution for each stimulus iteration, and a coefficient of variation $(\mathrm{CV})$ of spike counts in each bin across multiple renditions of the BOS was then calculated. The CV values for all bins during the stimulus were then averaged to obtain a mean CV of the stimulus-evoked response. A similar mean $\mathrm{CV}$ was calculated for a prestimulus baseline period equal in length to the stimulus duration, and this mean baseline $\mathrm{CV}$ was then subtracted from the mean stimulus-evoked CV to yield an estimate of the net mean stimulus-related CV or response CV. The response CV values of all LMAN neurons recorded in a single bird were averaged to generate a mean response CV value for each animal.

Histology. After each recording session, birds were deeply anesthetized with Equithesin and perfused transcardially with $0.9 \%$ saline for $\sim 3 \mathrm{~min}$, followed by $4 \%$ paraformaldehyde (PFA) in $25 \mathrm{~mm}$ sodium phosphate buffer for $30 \mathrm{~min}$. Brains were removed and postfixed in 4\% PFA with $30 \%$ sucrose overnight, blocked sagitally, and sectioned on a freezing microtome at $60 \mu \mathrm{m}$. Sections were processed with Avidin Alexafluor488 (Invitrogen, Eugene, OR); sections were incubated overnight at $4^{\circ} \mathrm{C}$ in a 1:500 dilution of Avidin Alexafluor- 488 and $0.4 \%$ Triton X-100 in 0.025 м PBS, rinsed four times for $20 \mathrm{~min}$ in PBS, mounted, and coverslipped). Neurobiotin-filled cells and biocytin-labeled extracellular recording sites were visualized using epifluorescence illumination. Sections were also treated with cresyl violet to identify outlines of LMAN structure and to confirm that the recorded cells and sites fell within these boundaries.

\section{Results}

We used quantitative methods to determine the amount and persistence of spectral distortion in song induced by vocal nerve section and developed an objective measure for determining whether or not an individual bird had undergone alterations in the temporal pattern of song (i.e., decrystallization). These analyses allowed us to determine that the spectral distortion of song after nerve section was immediate, pronounced, and persistent but that the song temporal pattern remained stable for at least 2 weeks after the surgery. We then assessed auditory selectivity of LMAN neurons in individual birds at relatively short ( $<2$ weeks) or longer ( $>3$ weeks) times after vocal nerve section, before or after distorted auditory feedback had triggered decrystallization.

\section{Spectral distortion of crystallized song after unilateral vocal nerve section}

Unilateral section of the vocal nerve in adult male zebra finches (see Materials and Methods) immediately triggered severe and persistent distortions in the spectral features of all birds' songs ( $n=51$ birds). Typical effects included a loss of frequency modulation within individual syllables, spectral "blurring" of harmonic stacks, and the replacement of tonal elements with broad harmonic stacks or noise (Fig. 1C,D) (the audio files of the songs shown in Fig. $1 C$ are provided with the supplemental material, available at www.jneurosci.org). We also noted more rarely that high narrowband notes found in some birds' motifs were dropped immediately after unilateral nerve section, presumably reflecting lateralized control (Goller and Cooper, 2004). These effects of syringeal paralysis on the acoustical features of song are consistent with the known role of the syringeal muscles in the rapid control of pitch and vocal filtering (Goller and Suthers, 1996).

To quantify the effects of vocal nerve section on song, we made within-bird comparisons of the song's spectral features before and at various times after nerve section using Sound Analysis-2 (Tchernichovski et al., 2000). The average spectral similarity scores (see Materials and Methods) between songs produced by the same bird before and after nerve section were low compared with within-bird comparisons made before nerve section (Fig. 2A) (pre, $86 \pm 3 \%$; post1, $31 \pm 4 \%$; post2, $31 \pm 8 \%$; post $3,38 \pm 5 \%$; df $=3 ; F=52 ; p<0.001$, one-way repeatedmeasures ANOVA; $p<0.01$, Fisher's post hoc test; $n=22$; pre vs post1, post2, and post3; unless stated otherwise, all values are expressed as mean \pm SEM). Indeed, the within-bird spectral similarity scores comparing pre- and post-nerve section songs at all times were as low as similarity scores measured between unrelated zebra finch songs recorded from our colony (mean spectral similarity of unrelated zebra finch songs, $28 \pm 5 \%$; df $=3 ; F=1$; $p=0.4$, one-way ANOVA for unrelated and post 1 , post 2 , and post3). Notably, the pronounced spectral distortion persisted without detectable recovery for as long as we tracked any of these birds (maximum up to $125 \mathrm{~d}$ ). Therefore, in all birds used in these experiments, unilateral section of the vocal nerve precipitated irreversible spectral distortion of their crystallized songs.

\section{Song decrystallization at longer times after vocal nerve section}

Our subjective impression was that the songs of all birds remained intact in their temporal structure (i.e., crystallized) for at least 2 weeks after vocal nerve section and decrystallized in a subset of birds over longer periods (i.e., $>3$ weeks) (Fig. 1B,C). Indeed, such delayed onset of song decrystallization in adult ze- 
A

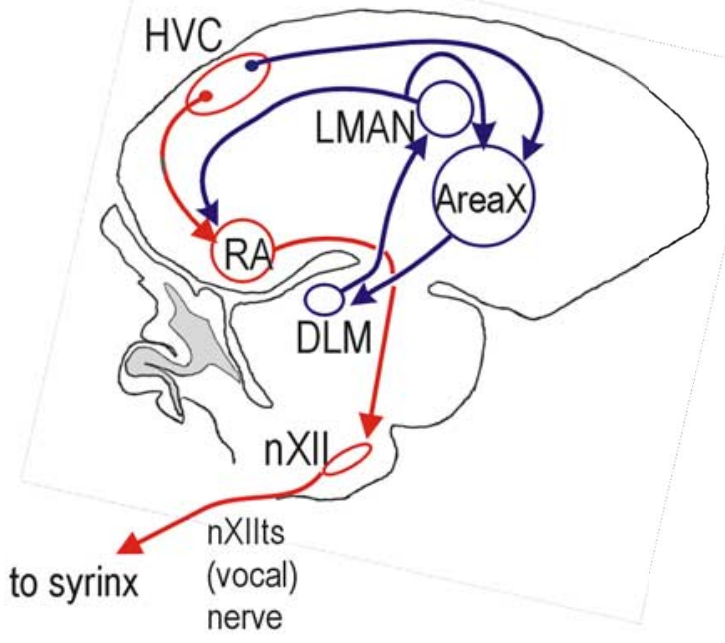

B

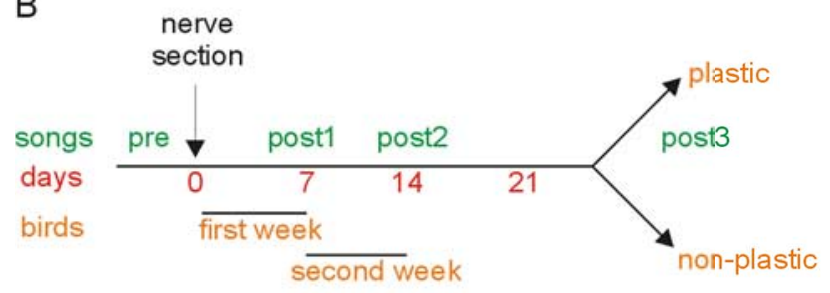

C
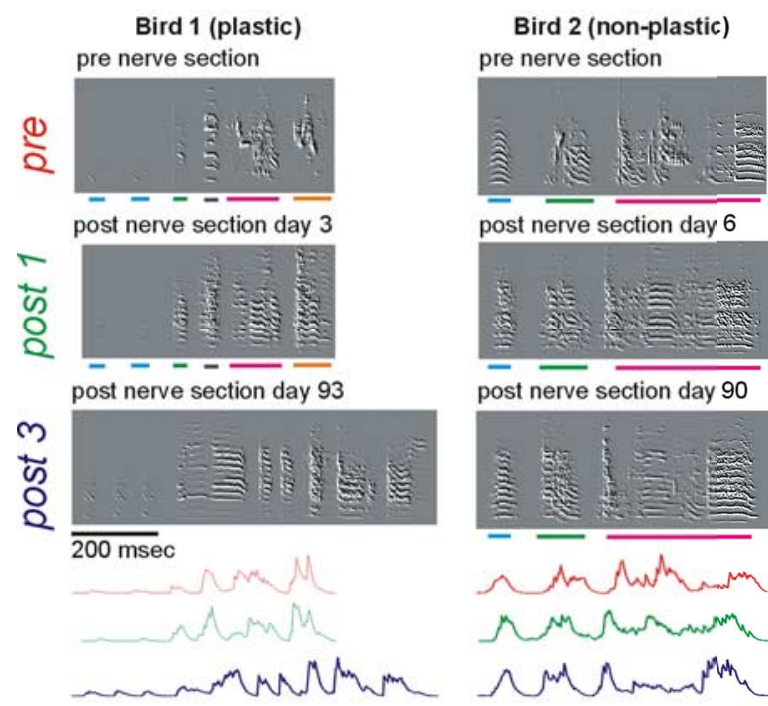

post nerve section day 6

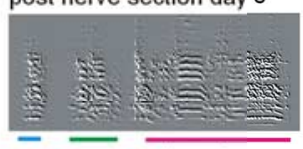

post nerve section day 90

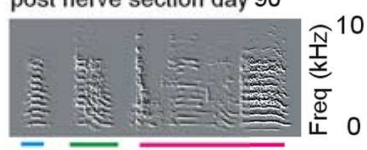

Non whingm

renchomemth $\wedge_{M m i n g}$

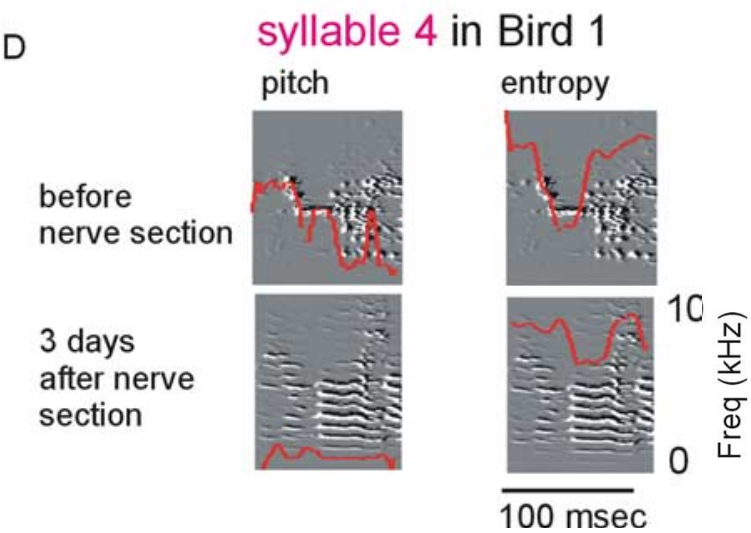

bra finches has been noted previously after vocal nerve section (Williams and McKibben, 1992), as well as after deafening (Nordeen and Nordeen, 1992) and superimposing delayed auditory feedback during singing (Leonardo and Konishi, 1999). However, to determine how auditory selectivity in LMAN changed relative to the onset of vocal plasticity, a more quantitative measure was needed for assessing whether and when song decrystallization had occurred after vocal nerve section. Therefore, we assessed decrystallization by measuring the stability of the amplitude envelope of the bird's song recorded at various times after vocal nerve section (Fig. 1C). We focused on the amplitude envelope of the song because it is primarily determined by expiratory activity (Wild et al., 1998), a feature that is controlled independently of the vocal nerve and thus spared by nerve section (Wild, 1993a,b, 1997; Suthers et al., 1999).

To quantify changes to the song amplitude envelope, we calculated a TI score for each bird. The TI score was calculated by cross-correlating the amplitude envelopes of a bird's songs (only single motifs, excluding introductory notes, were used for all song analysis) produced shortly after nerve section to those produced at longer intervals after nerve section (Fig. 2B) (see Materials and Methods). We also calculated a stereotypy score (essentially a within-bird, within-day TI score) by comparing amplitude envelopes of different bouts of songs recorded from a bird on a single day before nerve section. In all long-term birds, the stereotypy scores calculated before vocal nerve section were high (Fig. 2B) (within-bird, within-day TI score, $0.86 \pm 0.01 ; n=$ 22 ). We set an operationally defined threshold for song decrystallization as 2 SDs below this mean stereotypy score.

We found that the vast majority of second-week (15 of 17) and long-term (21 of 22) birds maintained TI scores above threshold for the first 2 weeks after nerve section (Fig. $2 B$, early) (data for only the 22 birds included in the long-term group are shown here). However, at longer times ( $>3$ weeks), the TI scores of a subset of birds ( $n=8$ of 22 ) fell below threshold, and these birds were deemed to have undergone song decrystallization. These eight birds with decrystallized songs were termed plastic birds, whereas the other 14 birds that maintained TI scores above threshold were termed non-plastic birds (Fig. $2 B$, late) (see supplemental Fig. 2, available at www.jneurosci.org as supplemental

$\leftarrow$

Figure 1. The effects of vocal nerve section on songs of adult male zebra finches. A, Simplified sagittal view of the song system showing the two major pathways controlling song plasticity and production. The song motor pathway (in red) is involved in the production of song. The AFP (in blue) is involved in juvenile and adult song plasticity. HVC, Proper name for the nucleus; RA, robust nucleus of arcopallium; nXII, hypoglossal nucleus, 12th cranial nerve nucleus; DLM, dorsolateral thalamic nucleus. $\boldsymbol{B}$, Timeline of the vocal nerve section experiments. After nerve section (day 0 ), adult male zebra finches were monitored for varying number of days, as indicated by the numbers in red. Depending on when electrophysiology was done and whether the birds underwent song decrystallization, birds were classified into four main categories, as indicated in orange. The songs recorded from a single bird at different times were designated as shown in green. C, Spectral derivative plots of songs before and after vocal nerve section in two adult male zebra finches. The colored bars below each plot identify individual syllables within a bird. Note the increased spectral distortion in all syllables shortly after nerve section in both birds. The large-scale temporal structure as defined by the order of syllables was maintained shortly after nerve section but underwent alterations at longer times in bird 1 (plastic), while remaining intact in bird 2 (non-plastic). The bottom panel shows smoothed and rectified amplitude waveforms of the songs recorded before nerve section (red), shortly after nerve section (green), and at longer times after nerve section (blue). In bird 1, the amplitude waveform at late times was markedly different from previous versions, whereas in bird 2, the original waveform was maintained. $\boldsymbol{D}$, (hanges in pitch and entropy of a single syllable (syllable 4 in bird 1) after vocal nerve section. The red traces depict the continuous pitch and entropy values of the syllable before and after vocal nerve section. Freq, Frequency. 
A

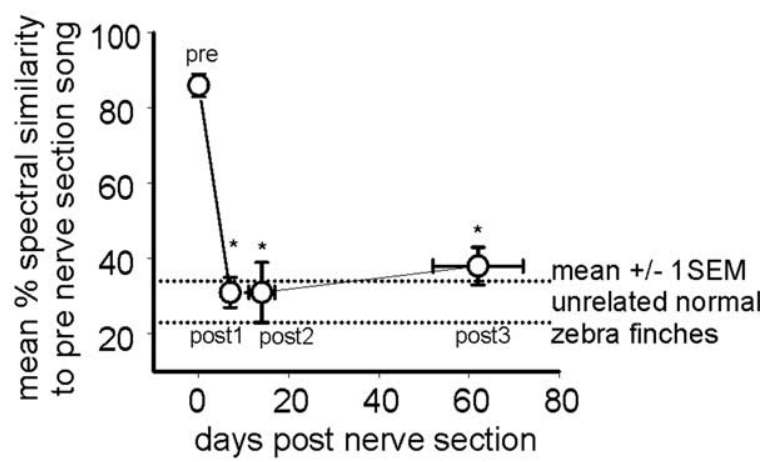

B

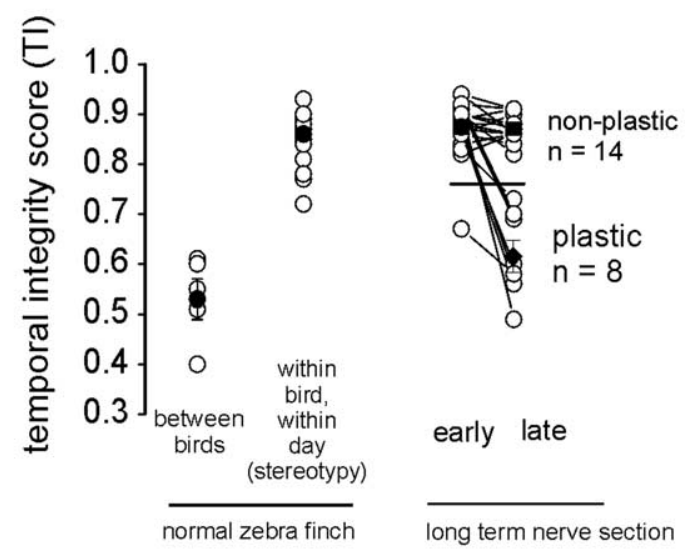

C

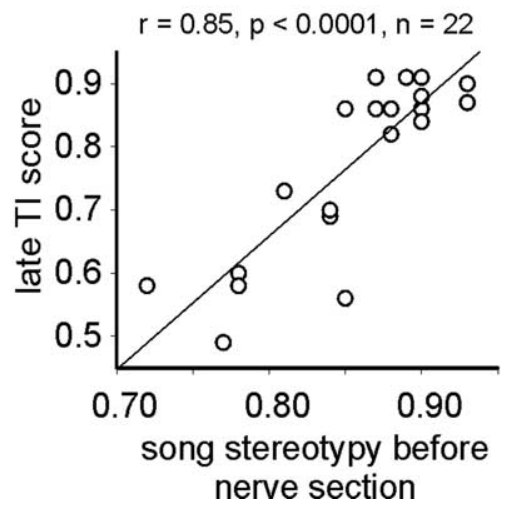

Figure 2. Quantification of the spectral and temporal changes in song after vocal nerve section. $A$, Mean percentage spectral similarity to pre-nerve section song ( $y$-axis, mean \pm SEM) at different intervals after nerve section ( $x$-axis, mean \pm SEM) for 22 long-term birds. Time point 0 (pre) represents the mean within-bird spectral similarity observed between multiple bouts of pre-nerve section songs. Time points denoted post1, post2, and post3 represent the average intervals after nerve section at which the first, the second, and the last song recordings were made (see Fig. $1 B$ ). Dotted lines denote mean \pm 1 SEM of spectral similarity between unrelated normal adult zebra finch songs recorded from our colony. $\boldsymbol{B}$, TI scores for songs of normal birds (left; between birds or within-bird, within-day comparison) and for songs of individual birds at early and late times after nerve section. Open circles, TI scores for individual birds; filled circles and error bars, mean \pm SEM for a given group. The mean late TI scores are reported separately for plastic (filled square) and non-plastic (filled diamond) birds. The horizontal line marks the mean pre-nerve section stereotypy score -2 SDs, below which song was operationally defined as decrystallized. In plastic birds, the late TI scores had decreased to a level approaching TI scores obtained between different zebra finch songs. In non-plastic birds, songs remained stable, as reflected in TI scores resembling stereotypy scores in normal adult zebra finches. C, The late TI scores strongly correlated with stereotypy scores measured before nerve section, indicating that birds with greater bout-to-bout song variability before nerve section were more likely to decrystallize their songs after vocal nerve section. material, for a detailed description of the distribution of TI scores at early and late times after nerve section). Most (seven of eight) plastic birds underwent song decrystallization between the second and third week after nerve section $(19 \pm 3 \mathrm{~d}$, mean \pm SEM $)$, whereas one bird had a TI score that fell below threshold during the first week. Because songs were typically recorded only at weekly intervals, it is possible that the time to decrystallization was slightly shorter than the mean value of $19 \mathrm{~d}$. The high TI scores in the non-plastic birds were indistinguishable from the stereotypy scores calculated before nerve section (Fig. $3 B)(p=$ 0.41 , paired $t$ test; $n=14$ ), whereas the TI scores in the plastic birds were indistinguishable from TI scores obtained by comparing the amplitude envelopes of songs of unrelated adult male zebra finches from our colony (i.e., "between-birds" TI scores) (Fig. $2 B)(p=0.33$, unpaired $t$ test; $n=8$, between-birds TI scores vs late TI scores in eight birds). Thus, by an operationally defined criterion, a subset of birds underwent song decrystallization 2-3 weeks after vocal nerve section, whereas the majority of birds maintained stable song patterns.

We wondered whether certain factors might account for why certain birds decrystallized their songs after vocal nerve section. We found no significant differences between plastic and nonplastic birds with respect to the mean age at the time of nerve section (plastic: $135 \pm 3$; range, $123-150 \mathrm{~d}$; non-plastic: $133 \pm 2$; range, $120-145 \mathrm{~d} ; p=0.7$ ) (see supplemental Fig. $3 C$, available at www.jneurosci.org as supplemental material) and the mean number of days after nerve section at which TI was assessed (plastic: $61.4 \pm 11.9$; range, $29-125 \mathrm{~d}$; non-plastic: $58.7 \pm 7.1$; range, 21-116 d; $p=0.8$; see supplemental Fig. $3 C$, available at www. jneurosci.org as supplemental material). We also found no correlation between the late TI scores and the age of the animal at the time of nerve section, the interval between nerve section and the final song recording used to calculate the late TI score, and the amount of spectral distortion caused by nerve section (see supplemental Fig. 3, available at www.jneurosci.org as supplemental material, and associated text for details). However, we detected a strong correlation between the song stereotypy score measured before nerve section and the late TI scores (Fig. $2 C)(r=0.85 ; p<$ $0.0001 ; n=22)$, suggesting that birds that sang more variable songs before nerve section were more likely to undergo song decrystallization after nerve section.

Given that plastic birds were more likely to sing less stereotyped songs before nerve section, we wondered whether they also produced less stereotyped songs after nerve section. In support of this idea, we found that the mean song stereotypy score of plastic birds was significantly lower than in non-plastic birds (plastic, $0.8 \pm 0.04$; non-plastic, $0.91 \pm 0.004$; mean \pm SEM; $p<0.0001$, $t$ test). However, a closer examination of song stereotypy revealed that half of the plastic birds $(n=4)$ had stereotypy scores comparable to the non-plastic birds, whereas the other four plastic birds had songs with low stereotypy (see supplemental Fig. 4, available at www.jneurosci.org as supplemental material, for examples of both types of songs). Thus, in some adult birds, song decrystallization after vocal nerve section may be accompanied by diminished song stereotypy.

\section{Estimating changes in LMAN auditory selectivity after vocal nerve section}

To test how the auditory selectivity of LMAN neurons changed after vocal nerve section, we recorded auditory responses of LMAN neurons in urethane-anesthetized zebra finches from four distinct behavioral groups: (1) during the first week after nerve section (first-week birds, $n=12$ ), (2) during the second week 
after nerve section (second-week birds, $n=15)$, (3) at $>3$ weeks after nerve section and without song decrystallization (non-plastic birds, $n=14$ ), and (4) at $>3$ weeks after nerve section and with song decrystallization (plastic birds, $n=8$ ). The latter two groups (groups 3 and 4) were formed from the ranks of the 22 birds previously used for the long-term behavioral analysis (Fig. $2 A, B$ ). The two short-term groups of birds (groups 1 and 2) consisted of two additional sets of adult male zebra finches that were also subjected to vocal nerve section. We confirmed that the spectral features of the songs of these two short-term group of birds were immediately and persistently distorted after vocal nerve section, whereas the temporal structures of these songs remained crystallized (for a detailed description, see supplemental Fig. 5, available at www.jneurosci.org as supplemental material).

We used in vivo intracellular and extracellular recordings to measure subthreshold and suprathreshold responses of LMAN neurons to auditory presentation (i.e., playback) of the BOS recorded before vocal nerve section (old BOS) and to the distorted song recorded the day before the electrophysiological recording session (new BOS). For all neurons/sites that exhibited significant responses to either stimulus, auditory selectivity was expressed using a $d^{\prime}$ metric (Green and Swets, 1966), wherein a positive d'newold value $\left(d^{\prime}{ }_{\mathrm{N}-\mathrm{O}}\right)$ signifies a response bias to the new BOS and a negative value signifies a response bias to the old BOS (see Materials and Methods). Intracellular and multiunit extracellular $d^{\prime}{ }_{\mathrm{N}-\mathrm{O}}$ values measured in the same birds did not significantly differ from one another $(p>0.05$, unpaired $t$ tests, for all birds with multiple single and multiunit data points) and therefore were pooled together to calculate mean per-bird $d^{\prime}{ }_{\mathrm{N}-\mathrm{O}}$ values (see supplemental Fig. 6, available at www.jneurosci.org as supplemental material, for a detailed description of all neurons and sites recorded).

\section{Shifts in LMAN auditory selectivity during the first 2 weeks after vocal nerve section}

We observed that the auditory selectivity of LMAN neurons in adult zebra finches was strongly selective for the old BOS during the first week after vocal nerve section. Electrophysiological recordings made during the first week (day of recording: $4.5 \pm 1.5$ after nerve section, mean $\pm \mathrm{SD}$; range, $2-7 \mathrm{~d}$ ) showed that LMAN neurons exhibited strong subthreshold and suprathreshold responses to the old BOS and responded only weakly or not at all to the new BOS (Fig. 3A). All birds examined during the first week after vocal nerve section exhibited negative mean $d^{\prime}{ }_{\mathrm{N}-\mathrm{O}}$ values at the suprathreshold level (for distribution of $d^{\prime}{ }_{\mathrm{N}-\mathrm{O}}$ values for all neurons see supplemental Fig. 6A, C, available at www.jneurosci.org as supplemental material). The overall mean suprathreshold $d^{\prime}{ }_{\mathrm{N}-\mathrm{O}}$ value for the group was significantly less than zero (Fig. $4 A$ ) (first week: mean $d^{\prime}{ }_{\mathrm{N}-\mathrm{O}}=-0.92 \pm 0.1 ; p<0.001 ; n=12$ ), indicating an overall preference for the old BOS at shorter inter-
B

post nerve section day 11
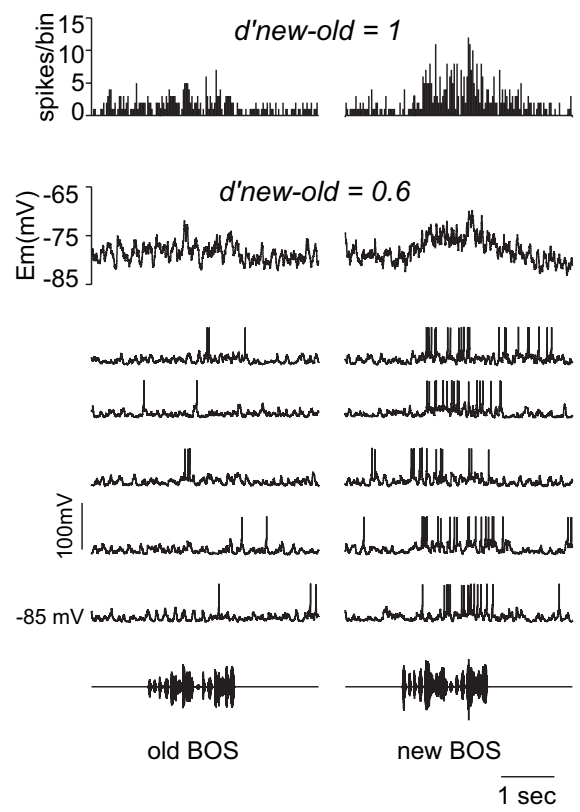

Figure 3. Subthreshold and suprathreshold auditory responses in LMAN neurons measured with in vivo intracellular recordings during the first and second weeks after vocal nerve section. $A$, Auditory responses of an LMAN neuron recorded intracellularly in an

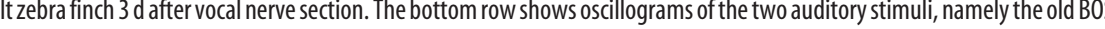
and suprathreshold activity were strongly evoked by the new, distorted BOS and only weakly evoked by the old BOS. For spectrograms of the two song stimuli, see supplemental Figure $2 A$ (available at www.jneurosci.org as supplemental material).

vals after vocal nerve section. This finding shows that shortly after nerve section, the distorted song is an ineffective stimulus for driving LMAN auditory responses, consistent with studies showing that BOS-selective neurons in an upstream song nucleus respond weakly or not at all to synthetic BOS variants in which spectral features were degraded (Theunissen and Doupe, 1998).

In contrast to recordings made from first-week birds, recordings made in second-week birds revealed that the new BOS could evoke strong responses in LMAN neurons in a subset of birds (Fig. $3 B$ ) (for a spectrogram of songs from this bird, see supplemental Fig. 5A, available at www.jneurosci.org as supplemental material). Five of 15 birds recorded during the second week after vocal nerve section exhibited mean positive $d^{\prime}{ }_{\mathrm{N}-\mathrm{O}}$ values, indicating preference for the new BOS (Fig. 4A). These positive $d^{\prime}{ }_{\mathrm{N}-\mathrm{O}}$ values represent an inversion of the LMAN response selectivity relative to the first week. The mean ${d^{\prime}}_{\mathrm{N}-\mathrm{O}}$ value for all secondweek birds was not significantly different from zero (second week: mean $\left.d^{\prime}{ }_{\mathrm{N}-\mathrm{O}}=-0.11 \pm 0.2 ; p=0.5 ; n=15\right)$, but was significantly different from that of first-week birds $(p=0.001$, unpaired $t$ test). The mean $d^{\prime}{ }_{\mathrm{N}-\mathrm{O}}$ value for the five birds with positive $d^{\prime}{ }_{\mathrm{N}-\mathrm{O}}$ was significantly different from zero $(0.66 \pm 0.15$; $p=0.01 ; n=5)$. These shifts in LMAN auditory selectivity occurred despite a maintained song temporal structure (i.e., in the absence of song decrystallization) (mean late TI score for all second-week birds was $0.88 \pm 0.01$; mean late TI score for only those 5 birds with positive $d^{\prime}{ }_{\mathrm{N}-\mathrm{O}}$ was $0.89 \pm 0.01$; see supplemental Fig. 5C, available at www.jneurosci.org as supplemental ma- 
A

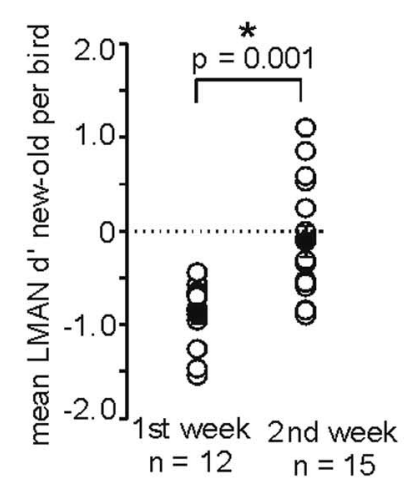

C

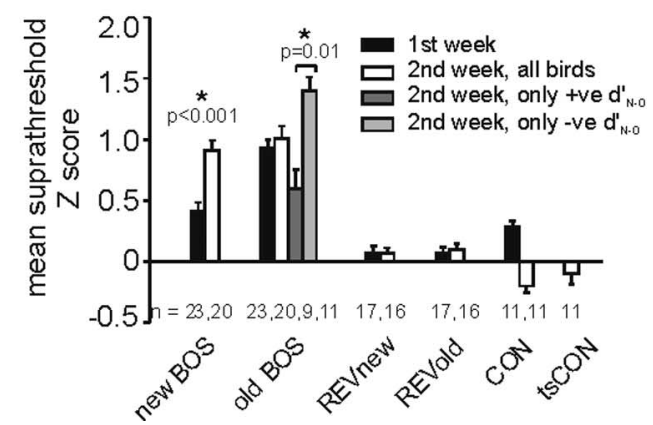

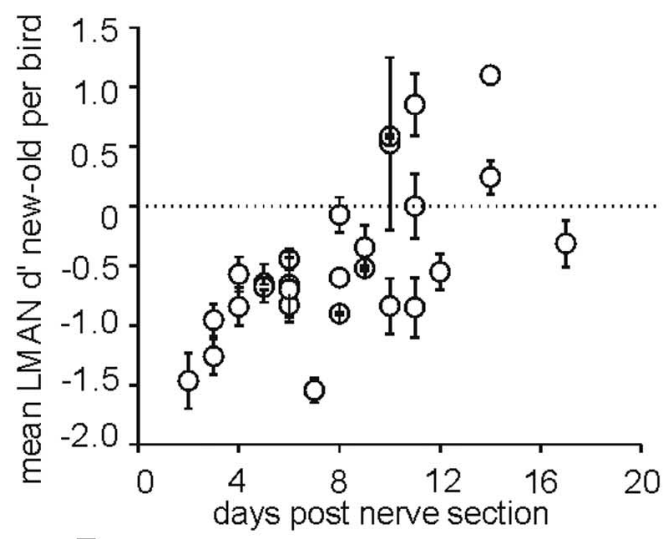

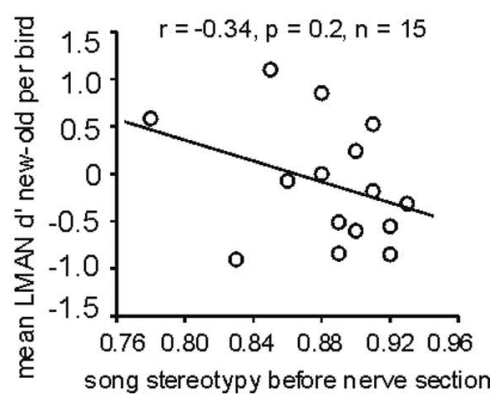

Figure 4. Shifts in auditory selectivity of LMAN neurons during the first 2 weeks after nerve section. $A$, Scatter plots of the mean LMAN $d^{\prime}{ }_{\mathrm{N}-\mathrm{O}}$ per bird during the first and second weeks after nerve section showed pronounced selectivity for the distorted new BOS in some second-week birds. Open circles, mean $d^{\prime}{ }_{\mathrm{N}-0}$ for individual birds (error bars not shown); filled circles, overall mean $d^{\prime}{ }_{N-0}$ for the group (error bars indicate SEM). $B$, Time course of the shifts in LMAN auditory selectivity during the first 2 weeks after nerve section. The mean LMAN $d^{\prime}{ }_{\mathrm{N}-\mathrm{O}}$ per bird was correlated with the interval between nerve section and electrophysiological recording $(r=0.6 ; p<0.0001 ; n=27)$. $C$, Mean suprathreshold response strengths of LMAN neurons, expressed as $Z$ scores, for a variety of auditory stimuli (labeled below the histograms) presented to the first-week and second-week birds. For second-week birds, the responses to the old BOS are also shown separately for birds with positive $(+v e)$ mean $d^{\prime}{ }_{\mathrm{N}-0}$ and negative $(-v e)$ mean $d^{\prime}{ }_{\mathrm{N}-0}$ values. Zscores were calculated only for LMAN neurons that showed detectable auditory responses to at least one of the test stimuli (see Materials and Methods). Numbers of neurons recorded for each stimulus are denoted below the histograms. $\boldsymbol{D}$, For second-week birds, the mean LMAN $d^{\prime}{ }_{\mathrm{N}-\mathrm{O}}$ per bird did not significantly correlate with song stereotypy measured before vocal nerve section. Error bars indicate SEM.

terial). Therefore, during the second week after vocal nerve section but in the absence of song decrystallization, there was a progressive shift toward greater responsiveness to the new BOS (e.g., see the cumulative distribution in supplemental Fig. $6 C$, available at www.jneurosci.org as supplemental material).

The time course over which LMAN selectivity shifted after nerve section was gradual and ultimately resulted in a subset of birds with positive $d^{\prime}{ }_{\mathrm{N}-\mathrm{O}}$ values (Fig. $4 B$ ). Over the first 2 weeks, we noted a positive correlation between mean $d^{\prime}{ }_{\mathrm{N}-\mathrm{O}}$ values for individual birds and the number of days after nerve section, indicating that LMAN auditory selectivity shifted gradually $(r=$ $0.6 ; p<0.001 ; n=27$ ). Furthermore, a positive correlation was maintained even when the five birds with positive mean $d^{\prime}{ }_{\mathrm{N}-\mathrm{O}}$ values were omitted from the sample $(r=0.5 ; p=0.01 ; n=22)$, indicating that even birds that did not invert their LMAN response preferences nevertheless underwent positive shifts in LMAN auditory selectivity. As suggested by the distribution of $d^{\prime}{ }_{\mathrm{N}-\mathrm{O}}$ values of individual LMAN neurons/sites (see supplemental Fig. $6 \mathrm{~A}$, available at www.jneurosci.org as supplemental material), the individual neurons/sites recorded in a single bird did not show wide disparity in $d^{\prime}{ }_{\mathrm{N}-\mathrm{O}}$ values. In summary, compared with the first-week birds, selectivity had shifted mark- edly in the LMAN of second-week birds, including a subset of birds $(n=5$ of 15$)$ in which LMAN neurons showed a response preference for the new, spectrally distorted BOS.

The positive shift in selectivity observed during the second week after vocal nerve section could involve a gain in response to the new BOS, a loss of response to the old BOS, or both. To distinguish between these possibilities, we expressed the suprathreshold response strengths as $Z$ scores (see Materials and Methods) for all neurons recorded. The mean response strength to the new BOS was significantly greater in the second week compared with the first week after vocal nerve section, indicating a gain in response to this newly experienced song (Fig. 4C) $(p<0.001$, unpaired $t$ test; $Z$ new BOS, first week vs second week). Overall, the mean response to the old BOS was unchanged between the first and second weeks. However, in the second-week birds exhibiting positive $d^{\prime}{ }_{\text {N-O }}$ values, the mean response to the old BOS was significantly diminished compared with second-week birds with negative d'new-old values (Fig. $4 C$ ) ( $p=0.01$, unpaired $t$ test). Therefore, the positive shift in LMAN selectivity that occurs during the second week involves a gain in response to the new BOS. Additionally, LMAN neurons in those birds that develop a response preference to the new BOS also showed diminished responsiveness to the old BOS.

One possibility is that the gain of responses to the new BOS after vocal nerve section reflects a nonselective increase in LMAN auditory responsiveness, similar to the expanded receptive field properties observed in primary sensory cortices in response to altered sensory experience (Merzenich et al., 1983, 1984; Bao et al., 2003). However, non-BOS stimuli, including other zebra finch songs (CON) and temporally reversed versions of the old and new BOS (REVold and REVnew, respectively), evoked little or no responses from LMAN neurons recorded during the first 2 weeks after nerve section (Fig. 4C). Another possibility is that LMAN neurons gain a general auditory responsiveness to songs with a high degree of spectral distortion. However, LMAN neurons recorded during the second week failed to respond to the songs of other zebra finches that had undergone vocal nerve section (Fig. $4 C$, tsCON). That LMAN neurons maintained highly selective response profiles even as they gained responses to new and distorted versions of the BOS was confirmed by the positive $d$ ' values for comparisons of the new BOS to REVnew, CON, and tsCON stimuli (d'new-REVnew $=1.48 \pm 0.3, n=17$; d'new-CON $=$ $1.7 \pm 0.4, n=11 ; \mathrm{d}^{\prime}$ new-tsCON $\left.=2.1 \pm 0.4, n=11\right)$. In the five birds with pronounced selectivity for the new BOS (i.e., positive $\left.d^{\prime}{ }_{\text {N-O }}\right)$, the mean selectivity for new BOS over these other song stimuli also remained high $\left(\mathrm{d}^{\prime}\right.$ new-REVnew $=1.6 \pm 0.6, n=6$; $\mathrm{d}^{\prime}$ new-CON $=1.95 \pm 0.7, n=5 ; \mathrm{d}^{\prime}$ new-tsCON $=2.3 \pm 0.9, n=$ 4 ), and these $d^{\prime}$ values were significantly higher than the mean 
$d^{\prime}{ }_{\mathrm{N}-\mathrm{O}}$ values in these five birds (mean $d^{\prime}{ }_{\mathrm{N}-\mathrm{O}}=0.66 \pm 0.15 ; p<0.05$, paired $t$ tests for all comparisons). Overall, the $d^{\prime}$ values measured in the birds with vocal nerve section were similar to or even greater than those measured in LMAN neurons of normal adult male zebra finches (Rosen and Mooney, 2000). Therefore, during the second week after vocal nerve section, LMAN neurons in some birds develop highly selective responses to the new, spectrally distorted BOS, rather than exhibiting a nonselective increase in auditory responsiveness.

Although the high TI scores in secondweek birds confirmed that the song temporal structure remained intact between the first and second weeks after vocal nerve section, these TI scores did not capture the within-day, bout-to-bout song variability. Thus, it remained plausible that songs produced by birds during the second week after vocal nerve section were less stereotyped, and this diminished stereotypy may have affected the shifts we observed in auditory selectivity during this period. If this were true, the stereotypy scores of the second-week birds should be low. Instead, the song stereotypy scores measured during the second week after nerve section were slightly higher than those measured before nerve section in the same birds, indicating that shifts in auditory selectivity were not attributable to increased song variability after vocal nerve section (mean stereotypy score, second week: after nerve section, $0.91 \pm 0.003$; before nerve section, $0.88 \pm 0.01$; mean \pm SEM; $p=0.02$, paired $t$ test; $n=15$ ). Furthermore, there was no significant difference in song stereotypy between the second-week birds with positive $d^{\prime}{ }_{\mathrm{N}-\mathrm{O}}$ values and those with negative $d^{\prime}{ }_{\mathrm{N}-\mathrm{O}}$ values (positive $d^{\prime}{ }_{\mathrm{N}-\mathrm{O}}$ birds, $0.91 \pm 0.007$; negative $d^{\prime}{ }_{\mathrm{N}-\mathrm{O}}$ birds, $0.91 \pm 0.003$; mean \pm SEM; $p=0.4, t$ test). Another possibility is that the shift in auditory selectivity observed during the second week correlated with greater pre-existing (i.e., before nerve section) song variability. Although there was a weak negative correlation between the mean $d^{\prime}{ }_{\mathrm{N}-\mathrm{O}}$ values measured in second-week birds and the stereotypy scores measured in these same birds before vocal nerve section, this correlation was not significant (Fig. 4D) $(r=-0.34 ; p=0.21 ; n=15)$. In addition, the mean pre-nerve section stereotypy scores for the second-week birds with positive $d^{\prime}{ }_{\mathrm{N}-\mathrm{O}}$ values and those with negative $d^{\prime}{ }_{\mathrm{N}-\mathrm{O}}$ values did not significantly differ (positive $d^{\prime}{ }_{\mathrm{N}-\mathrm{O}}$ birds, $0.86 \pm 0.02$; negative $d^{\prime}{ }_{\mathrm{N}-\mathrm{O}}$ birds, $0.89 \pm 0.01$; mean \pm SEM; $p=0.2$, $t$ test). Therefore, the shifts in LMAN auditory selectivity during the second week after vocal nerve section occurred in the absence of song decrystallization and despite a high degree of song stereotypy (see supplemental Fig. $5 A, C$, available at www.jneurosci.org as supplemental material).

\section{Shifts in LMAN auditory selectivity at longer times after nerve section}

During the second week after vocal nerve section, LMAN neurons in some birds exhibited positive $d^{\prime}{ }_{\mathrm{N}-\mathrm{O}}$ values, whereas LMAN neurons in other birds exhibited negative $d^{\prime}{ }_{\mathrm{N}-\mathrm{O}}$ values. At the behavioral level, distorted auditory feedback acted over
B

non-plastic (38 days post nerve section)
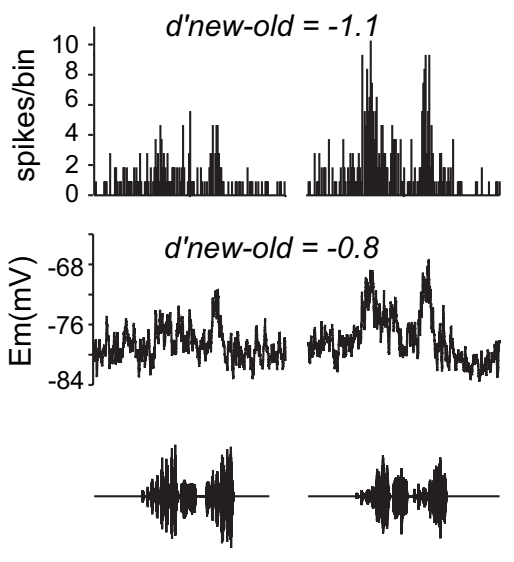

new BOS

old BOS longer times ( $>3$ weeks) to trigger song decrystallization in a subset of birds. One possibility is that these bimodal outcomes of vocal nerve section at the neuronal and behavioral levels are related. More specifically, the maintained neural capacity in adult birds to gain auditory response selectivity to new versions of the BOS could be correlated with the behavioral capacity of these birds to undergo song decrystallization. If this model is correct, then LMAN neurons in plastic birds should display selectivity for the new, decrystallized BOS.

Consistent with this idea, almost all (44 of 47) LMAN neurons and sites in plastic birds $(n=8)$ showed positive $d^{\prime}{ }_{\text {N-O }}$ values, indicating an auditory preference for the new BOS (for distribution of $d^{\prime}{ }_{\mathrm{N}-\mathrm{O}}$ values for all neurons/sites, see supplemental Fig. $6 B, C$, available at www.jneurosci.org as supplemental material). A majority of neurons in plastic birds responded strongly at both the subthreshold and suprathreshold levels to the new, temporally reorganized version of the BOS, whereas the old (i.e., recorded before nerve section) BOS elicited little or no response (Fig. 5A). The mean $d^{\prime}{ }_{\text {N-O }}$ values for all eight plastic birds were positive, and the overall mean for the group was significantly different from zero (Fig. $6 A$ ) (plastic birds, $d^{\prime}{ }_{\mathrm{N}-\mathrm{O}}=0.8 \pm 0.2$; $p=0.001 ; n=8$ ), indicating an overall auditory selectivity for the new BOS. Indeed, an analysis of suprathreshold responses to a variety of song stimuli showed that the new BOS was the most effective stimulus in plastic birds, whereas the old BOS evoked a significantly weaker response (Fig. $6 B)(p<0.001$, paired $t$ test; $n=28$; Z scores for new BOS vs old BOS). Importantly, versions of the BOS recorded shortly after vocal nerve section but before song decrystallization (tsBOSold), as well as REVnew, REVold, and $\mathrm{CON}$, were ineffective at driving LMAN responses in plastic birds (Fig. 6B). Notably, the spectrally distorted but temporally intact song could drive responses in LMAN of some of the second-week birds, as described previously. Therefore, LMAN 
A

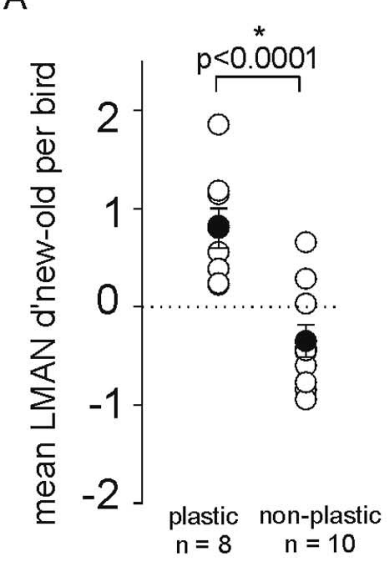

C

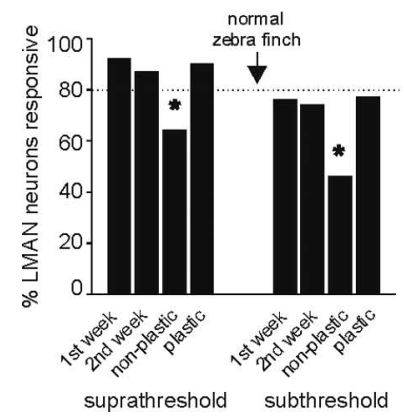

B

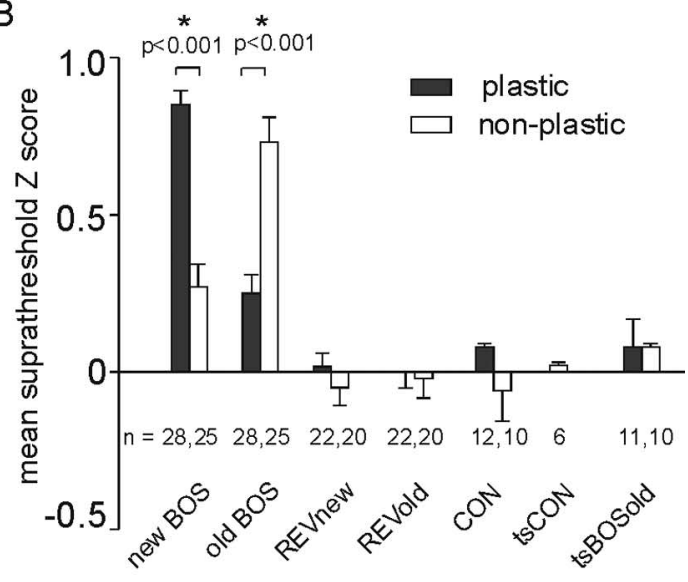

$\mathrm{D}$

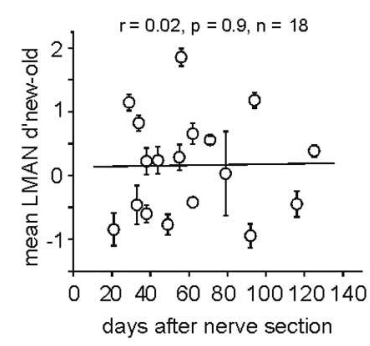

$\mathrm{E}$

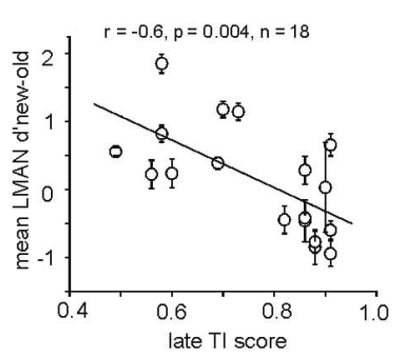

Figure 6. Quantification of auditory selectivity and responsiveness in LMAN neurons of plastic and non-plastic birds. A, Scatter plot of mean LMAN $d^{\prime}{ }_{\mathrm{N}-0}$ (open circles; per bird) showed that auditory responses in plastic birds were significantly biased to the new BOS compared with the non-plastic birds. Filled circles and error bars denote mean and SEM for the two groups. $\boldsymbol{B}$, Mean suprathreshold responses of LMAN neurons for various auditory stimuli, expressed as Zscores, indicate that the new BOS was the most effective stimulus in plastic birds, whereas the old BOS was the most effective stimulus in non-plastic birds. Other song stimuli were ineffective in eliciting auditory responses in LMAN neurons of both plastic and non-plastic birds. The number of neurons recorded for each stimulus is denoted at the bottom of each histogram. Error bars indicate SEM. C, At both the subthreshold and suprathreshold levels, significantly fewer LMAN neurons in non-plastic birds responded to any auditory stimulus relative to the number of responsive LMAN neurons in other experimental groups. The dotted line denotes the proportion of LMAN neurons with auditory activity in normal adult zebra finches (Rosen and Mooney, 2000). D, The mean LMAN $d^{\prime}{ }_{N-0}$ values per bird did not correlate with the time interval between nerve section and electrophysiological recording. $\boldsymbol{E}$, The mean LMAN $d^{\prime}{ }_{N-0}$ values per bird negatively correlated with late TI scores, indicating that birds that decrystallized their songs contained LMAN neurons with a response preference for the new BOS.

auditory selectivity in plastic birds tracks the current version of the BOS without involving a general increase in auditory responsiveness to other stimuli.

In contrast to LMAN neurons in plastic birds, the majority of LMAN neurons in the non-plastic birds either lacked auditory responses altogether or maintained selectivity for the old BOS (Fig. 5B). Notably, we failed to detect any responses to old or new versions of the BOS in several non-plastic birds $(n=4)$ (see supplemental Fig. $6 B$, available at www.jneurosci.org as supplemental material). In the rest of the non-plastic birds $(n=10)$ with at least some responsive LMAN neurons, the fraction of LMAN neurons that exhibited any subthreshold or suprathreshold responses (subthreshold, 18 of 39; suprathreshold, 25 of 39) to old or new versions of the BOS was significantly smaller than the fraction of responsive LMAN neurons encountered in either plastic birds, birds within the first 2 weeks after nerve section, or normal adult male zebra finches $(\sim 80 \%)$ (Rosen and Mooney, 2000) (Fig. 6C) ( $\chi^{2}$ tests for both subthreshold and suprathreshold; $p<0.001$ for non-plastic; $p>0.05$ for first-week, secondweek, and plastic birds compared with normal zebra finches). Moreover, in the 10 non-plastic birds with at least some respon-

sive LMAN neurons/sites, the old BOS was the most effective stimulus for driving suprathreshold responses in LMAN neurons (Fig. 6B). Among these 10 birds, the mean $d^{\prime}{ }_{\mathrm{N}-\mathrm{O}}$ values were negative in seven birds, positive in two birds, and very close to zero in the other (Fig. 6A) (note that nonresponsive cells were not included to calculate these mean $d^{\prime}{ }_{\mathrm{N}-\mathrm{O}}$ values). The mean $d^{\prime}{ }_{\mathrm{N}-\mathrm{O}}$ value in all non-plastic birds with some responsive neurons was not significantly different from zero (Fig. 6A) (nonplastic birds: $d^{\prime}{ }_{\mathrm{N}-\mathrm{O}}=-0.35 \pm 0.16 ; p=$ $0.09 ; n=10)$ and was significantly lower than the mean $d^{\prime}{ }_{\mathrm{N}-\mathrm{O}}$ value measured in plastic birds ( $p<0.001$, unpaired $t$ test). However, the mean $d^{\prime}{ }_{\mathrm{N}-\mathrm{O}}$ values in nonplastic birds were significantly and positively shifted relative to the first-week birds (first week, $d^{\prime}{ }_{\mathrm{N}-\mathrm{O}}=-0.92 \pm 0.1 ; p=$ 0.01 , unpaired $t$ test; first week vs nonplastic). Therefore, at long times after nerve section in the absence of song decrystallization, we observed either a loss of auditory responsiveness in LMAN or moderate shifts in selectivity.

One potential caveat is that differences in LMAN selectivity we observed between plastic and non-plastic birds might have been attributable to different durations over which these two groups experienced distorted feedback, rather than their distinct behavioral outcomes. However, the mean $d^{\prime}{ }_{\mathrm{N}-\mathrm{O}}$ values in individual birds did not correlate with the interval between vocal nerve section and electrophysiological recording (Fig. $6 D)(r=0.02 ; p=0.9 ; n=$ 18). The mean intervals between nerve section and electrophysiological recordings for the plastic and non-plastic birds were also not significantly different (see supplemental Fig. $3 C$, available at www. jneurosci.org as supplemental material), indicating that the differences in selectivity between plastic and non-plastic birds could not be attributed to differences in the duration of distorted feedback experience. Alternatively, if the differences in selectivity were related to the presence or absence of song decrystallization, birds with lower TI scores (i.e., plastic birds) should have higher $d^{\prime}{ }_{\mathrm{N}-\mathrm{O}}$ values. Indeed, such a negative correlation was observed (Fig. 6E) $(r=-0.6 ; p=0.004 ; n=18)$. Thus, the differences in auditory plasticity observed between plastic and non-plastic birds correlated strongly with the distinct behavioral outcomes after vocal nerve section.

\section{Loss of subthreshold responsiveness to the original song after} song decrystallization

In some sensorimotor systems, altered sensory experience can shift suprathreshold response patterns, whereas synapses conveying information about previously preferred stimuli are anatomically maintained but functionally suppressed, at least at the suprathreshold level (Zheng and Knudsen, 1999; Linkenhoker et al., 2005). We wondered whether LMAN neurons in plastic birds, while showing suprathreshold selectivity for the new BOS, might 
still exhibit purely subthreshold responses to the previously preferred stimulus, namely the old BOS. To test this idea, we analyzed the subthreshold depolarizing responses of LMAN neurons in plastic birds with intracellular recording methods. In plastic birds, LMAN neurons typically exhibited strong depolarizing membrane potential responses to the playback of new BOS but not the old BOS (as exemplified in the neuron shown in Fig. 5A). For individual neurons recorded in plastic birds, the subthreshold and suprathreshold responses to the old BOS were not significantly different, indicating that subthreshold responses to the old BOS were not maintained in the absence of suprathreshold responses (Fig. 7A) ( $p=0.5 ; n=31$, paired $t$ test; subthreshold vs suprathreshold $Z$ scores). A further indication that responses to the old BOS were not maintained synaptically is that LMAN neurons in plastic birds were equally selective for the new BOS at the subthreshold and the suprathreshold levels (Fig. 7C) $(p=0.08$, paired $t$ test; $n=22$; subthreshold vs suprathreshold $d^{\prime}{ }_{\mathrm{N}-\mathrm{O}}$ values). Therefore, in plastic birds, there was a loss of synaptic responsiveness in LMAN to the old BOS (Fig. 7D) (mean subthreshold $Z$ score to old BOS: first week, $1.1 \pm 0.1$; plastic, $0.2 \pm 0.08 ; p<0.001$, unpaired $t$ test).

Conversely, only subthreshold responses to the new BOS may have been evoked in LMAN neurons of non-plastic birds. However, pairwise comparisons within individual neurons revealed that this was not the case: subthreshold and suprathreshold responses to the new BOS in non-plastic birds were similar (Fig. $7 B$ ) ( $p=0.07$, paired $t$ test; $n=54$; subthreshold vs suprathreshold $Z$ scores). Additionally, LMAN neurons in non-plastic birds were equally selective for the new BOS compared with the old BOS at the subthreshold and suprathreshold levels (Fig. 7C) ( $p=$ 0.9 , paired $t$ test; $n=16$; subthreshold vs suprathreshold $d^{\prime}{ }_{\mathrm{N}-\mathrm{O}}$ values). Finally, in non-plastic birds, the mean subthreshold response $Z$ scores to the new BOS were indistinguishable from those recorded during the first week after vocal nerve section (Fig. 7D) (mean subthreshold $Z$ score to new BOS: first week, $0.35 \pm$ 0.1 ; non-plastic, $0.31 \pm 0.1 ; p=0.5$, unpaired $t$ test). Therefore, LMAN neurons of non-plastic birds lacked responses to the new BOS at both the subthreshold and suprathreshold levels and lacked latent (i.e., only subthreshold) responses to the spectrally distorted BOS.

\section{Do LMAN auditory response properties correlate with song stereotypy?}

We noted that birds that undergo decrystallization exhibit strong shifts in auditory selectivity in LMAN. We also observed that birds with lower song stereotypy were more likely to undergo decrystallization after vocal nerve section. These observations prompted us to ask whether the auditory response properties of
B
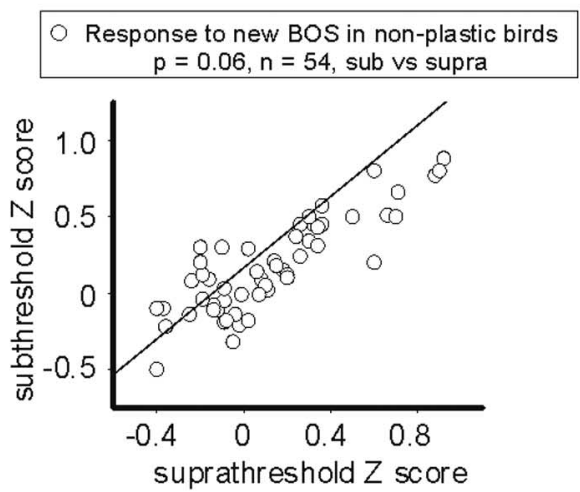

D

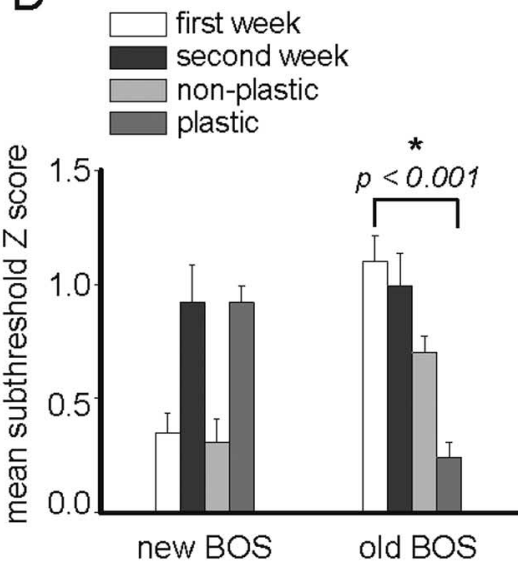

new BOS

Figure 7. Comparison of subthreshold and suprathreshold changes in LMAN auditory responses after nerve section. $\boldsymbol{A}$, Sub列 comparisons, only LMAN neurons that responded at both the subthreshold and suprathreshold levels were considered. $\boldsymbol{D}$, Mean not in the non-plastic birds. There was a drastic reduction in subthreshold responses to the old BOS in plastic birds, signifying a loss of synaptic drive to LMAN neurons in response to this stimulus. Error bars indicate SEM. sub, Subthreshold; supra, suprathreshold.

LMAN neurons in normal adult birds differed as a function of song stereotypy. To address this issue, we searched for correlations between auditory response properties of LMAN neurons used in a previously published study (Rosen and Mooney, 2000) and the stereotypy of the songs of the birds from which these electrophysiological data were obtained. In addition to response strength and selectivity, we also characterized the temporal variability of LMAN auditory responses (see Materials and Methods), because variability in singing-related activity of LMAN neurons has been shown to correlate with acoustic variability of song (Kao et al., 2005). These analyses revealed no correlation between song stereotypy and these three metrics of LMAN auditory responses (Fig. $8 A-C$ ). Because songs were always recorded in the presence of a female bird, and such directed songs exhibit less acoustic variability compared with undirected songs (Kao et al., 2005; Kao and Brainard, 2006), it remains possible that LMAN auditory properties correlate with song variability only when greater variability is manifested in song structure. To test this possibility, we correlated LMAN response variability with song variability in juvenile birds, which sing highly variable songs. However, we 
A

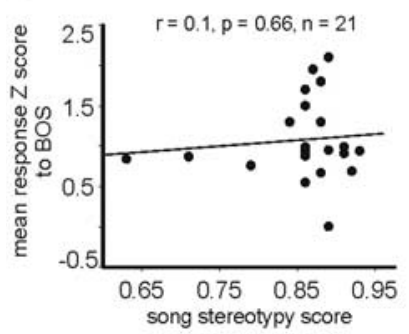

C

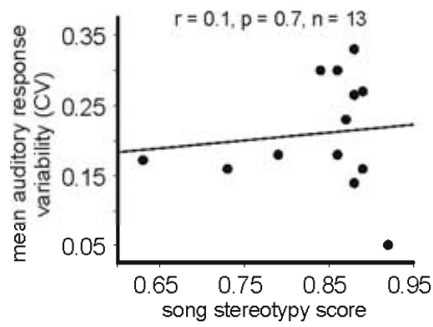

B

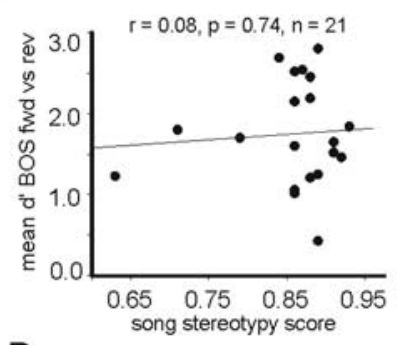

D

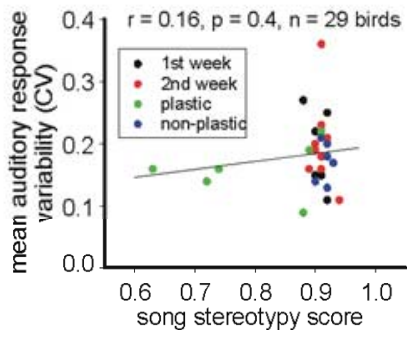

Figure 8. Correlational analysis of LMAN auditory response properties and song stereotypy scores in normal $(\boldsymbol{A}-\boldsymbol{C})$ and nerve-sectioned $(\boldsymbol{D})$ birds. $\boldsymbol{A}$, In normal adults, the mean LMAN auditory response strength per bird did not correlate with song stereotypy scores. Response strength was measured to playback of the BOS and expressed as $Z$ score. $\boldsymbol{B}$, In normal adults, the mean selectivity ( $d$ ' per bird) of LMAN auditory responses to playback of forward (fwd) versus reverse (rev) BOS did not correlate with song stereotypy scores. C, In normal adults, the mean temporal variability per bird of LMAN auditory responses to playback of BOS did not correlate with song stereotypy scores. $\boldsymbol{D}$, In nerve-sectioned birds of all four categories, the mean temporal variability per bird of LMAN auditory responses did not correlate with the song stereotypy measured just before the time of electrophysiological recording.

failed to detect a correlation between LMAN response variability and song stereotypy in a set of five juvenile birds singing variable songs (for details, see supplemental Fig. 7A, available at www.jneurosci.org as supplemental material). Therefore, in normal juvenile and adult male zebra finches, none of the auditory response properties of LMAN neurons we characterized correlated with song stereotypy.

We also analyzed the temporal variability of LMAN auditory activity in the nerve-sectioned birds, because some birds subjected to this manipulation could show increased levels of song variability (i.e., four of the plastic birds). As with normal adult birds, we found no correlation between song stereotypy and the temporal variability of LMAN auditory responses in nervesectioned birds (Fig. 8D). However, we did note that secondweek birds that had developed positive $d^{\prime}{ }_{\mathrm{N}-\mathrm{O}}$ values also exhibited greater temporal variability in their auditory responses to this stimulus, despite maintaining highly stereotyped songs (see supplemental Fig. $7 B$, available at www.jneurosci.org as supplemental material).

\section{Discussion}

\section{LMAN auditory plasticity is not critical period limited}

Zebra finches learn to sing during a juvenile critical period (Immelmann, 1969; Price, 1979; Eales, 1985), then maintain their crystallized songs via auditory feedback (Nordeen and Nordeen, 1992; Williams and McKibben, 1992; Leonardo and Konishi, 1999). In sensorimotor areas for singing and song learning, highly selective auditory responses to the BOS develop in parallel with song learning (Doupe, 1997; Solis and Doupe, 1997). One idea is that BOS selectivity in areas important to vocal plasticity, including LMAN, becomes fixed after crystallization, serving as a

referent for song maintenance. Instead, we found that LMAN auditory selectivity in some adult zebra finches could shift to spectrally distorted songs after vocal nerve section. This constitutes the first example of electrophysiological plasticity in the song system beyond the closure of the critical period for song learning, refuting the idea that the closure of the critical period for song learning may stem from a complete loss of neural plasticity in LMAN. Our results also indicate that neural plasticity in the adult songbird is not restricted to premotor areas directly controlling song but extends to a basal ganglia pathway necessary to audition-dependent song plasticity.

\section{In adults, LMAN neurons can develop selectivity for spectrally distorted songs}

Juvenile zebra finches subjected to vocal nerve section mature into adults that sing poor tutor song imitations and lack auditory responses in LMAN, although LMAN auditory responses selective for the distorted BOS could be recorded during sensorimotor learning in these birds (Solis and Doupe, 1999, 2000). These findings raise the possibility that the robust and selective auditory responses of LMAN neurons in the adult zebra finch reflect a good match between the crystallized BOS and the tutor song. One prediction of this model is that LMAN auditory responses in normal adults will diminish when the BOS deviates from its crystallized form. We found partial support for this view in the diminished proportion of auditory responsive LMAN neurons in non-plastic birds. However, the proportion of auditoryresponsive LMAN neurons in second-week and plastic birds was similar to normal birds, although songs of second-week, plastic, and non-plastic birds were equally distorted (supplemental Fig. $3 E$, available at www.jneurosci.org as supplemental material). Furthermore, many LMAN neurons in second-week and plastic birds gained responses to the spectrally distorted BOS. These findings are difficult to reconcile with a matching hypothesis. Instead, in adult birds, LMAN neurons can gain selectivity for severely distorted songs presumably less well matched to the tutor song than the originally crystallized BOS.

\section{LMAN auditory plasticity involves a gain in highly selective responses}

The precision with which L MAN selectivity shifts in the adult was most evident in plastic birds, wherein LMAN neurons responded to the current, decrystallized BOS but not to spectrally distorted versions of the BOS produced before decrystallization. This selective shift stands in sharp contrast to examples of aberrant feedback-induced reorganization in primary sensory cortices, which typically involve expanded receptive field structures. After denervation or digit amputation in monkeys, neuronal receptive fields in primary somatosensory cortex representing the remaining digits expand (Merzenich et al., 1983, 1984). Distorted auditory experience attributable to noise rearing in rats and vocal denervation in marmosets also leads to expanded receptive fields in primary auditory cortex (Bao et al., 2003; Cheung et al., 2005). In contrast, we found that LMAN neurons in the adult can gain responses specific to the current BOS, while losing auditory responses to previously preferred stimuli.

\section{Are shifts in LMAN selectivity due to auditory feedback?}

Our results hint that auditory feedback shapes BOS selectivity of LMAN neurons in adult zebra finches. However, most nonplastic birds failed to show pronounced shifts in LMAN selectivity, raising the possibility that feedback acts with other factors to shape LMAN auditory selectivity. In addition to their BOS- 
evoked auditory responses, LMAN neurons display singingrelated activity thought to be motor corollary discharge emanating from the telencephalic song nucleus HVC (Hessler and Doupe, 1999; Leonardo, 2004). Moreover, corollary discharge in the AFP has been hypothesized to be a motor-based prediction of the expected auditory feedback, generated by interactions between the song motor program and real auditory feedback (Troyer and Doupe, 2000). Although the high TI and stereotypy scores during the second week after nerve section indicated a stable song motor program, we cannot exclude the possibility that subtle changes to corollary discharge in LMAN occurred before decrystallization. Thus, in nerve-sectioned birds undergoing decrystallization, changes in this estimated feedback signal, rather than or in addition to auditory feedback, might have contributed to shifts in LMAN selectivity. According to this view, corollary discharge in the AFP of non-plastic birds is insensitive to recalibration by distorted feedback, consequently failing to alter LMAN auditory selectivity. In contrast, experience of chronically distorted feedback in plastic birds leads to recalibration of the corollary discharge, ultimately altering selectivity within LMAN. Nevertheless, even in this scenario, shifts in LMAN auditory selectivity are indirectly shaped by feedback.

Although both auditory and motor-related activity may shape LMAN auditory responses, we think it unlikely that the auditory response properties of LMAN neurons simply mirror their motor-related properties. Microstimulation and chronic recording studies indicate that song variability is driven by variable patterns of singing-related activity in LMAN neurons (Kao et al., 2005; Olveczky et al., 2005). If auditory and motor properties of LMAN neurons were tightly coupled, then features of their auditory responses should correlate with motor variability. However, we found that neither the strength nor selectivity of LMAN auditory responses correlated with song variability (i.e., diminished song stereotypy). Furthermore, we found no correlation between auditory response variability and song variability. Finally, in the second week after vocal nerve section, we saw that auditory selectivity and auditory response variability of LMAN neurons could change in the absence of detectable alterations to the song temporal pattern and despite high song stereotypy. These results suggest that auditory response properties in LMAN do not simply reflect the state of song motor activity and point to the importance of auditory experience in affecting the alterations in auditory selectivity we observed in adult nerve-sectioned birds.

\section{Is LMAN auditory plasticity causally linked to song decrystallization?}

We noted that LMAN auditory plasticity strongly correlated with song decrystallization: all plastic birds ( 8 of 8 ) contained LMAN neurons selective for the decrystallized new BOS, whereas half ( 7 of 14) of the non-plastic birds contained LMAN neurons selective for the old BOS. One possibility is that auditory plasticity in LMAN and vocal plasticity are two independent processes, possibly regulated by a common factor. Adult zebra finches with lower blood testosterone levels are more prone to decrystallize their songs after vocal nerve section (Williams et al., 2003), suggesting that androgen levels, or related factors, may independently regulate LMAN auditory plasticity and song decrystallization. Another possibility is that a gain in selective responses in LMAN to the spectrally distorted new BOS is a necessary precondition for decrystallization. This idea gains support from the observation that the fraction of second-week birds with pronounced LMAN auditory selectivity to the distorted BOS (5 of
15) closely resembled the fraction of birds that underwent decrystallization ( 8 of 22).

How might altered auditory properties in LMAN contribute to song plasticity? If the AFP conveys auditory feedback during singing, then altered auditory response properties could eventually contribute to changes in motor activity in LMAN, paving the way for song plasticity. On the one hand, we provide evidence that auditory plasticity in LMAN and motor plasticity can be dissociated in time, suggesting any such interactions are weak and slow. If auditory responses of LMAN are evoked only sporadically during singing, it may explain why a previous study failed to detect short-term effects of distorted auditory feedback on singing-related LMAN activity in adult zebra finches (Leonardo, 2004) and why decrystallization of adult zebra finch song, whether induced by nerve section, deafening, or delayed auditory feedback, is a slow process (Nordeen and Nordeen, 1992; Williams and McKibben, 1992; Leonardo and Konishi, 1999).

\section{Conclusions}

The capacity for auditory plasticity in the AFP of the adult songbird suggests that BOS-selective responses are not indelibly imprinted in the brain after the closure of the critical period for song learning and refutes the idea that a good match between the BOS and the tutor song is necessary for the expression of LMAN auditory responses beyond song crystallization. Moreover, the finding that shifts in auditory selectivity correlate strongly with song decrystallization raises the possibility that such auditory plasticity in LMAN links auditory feedback and song decrystallization. Finally, after a lower motor neuron injury, sensory responses were remodeled in the AFP. Because the AFP is necessary to central reorganization of the song motor program (Williams and Mehta, 1999; Brainard and Doupe, 2000a) and bears homologies to mammalian basal ganglia-forebrain pathways (Luo and Perkel, 1999; Farries and Perkel, 2000, 2002; Doupe et al., 2005), these findings may inform ideas about how basal ganglia pathways in the human contribute to pathology and recovery of complex motor skills after lower motor neuron injuries (Marsden and Rothwell, 1987; Schoenfeld et al., 2005; Tessitore et al., 2006).

\section{References}

Bao S, Chang EF, Davis JD, Gobeske KT, Merzenich MM (2003) Progressive degradation and subsequent refinement of acoustic representations in the adult auditory cortex. J Neurosci 23:10765-10775.

Brainard MS, Doupe AJ (2000a) Interruption of a basal ganglia-forebrain circuit prevents plasticity of learned vocalizations. Nature 404:762-766.

Brainard MS, Doupe AJ (2000b) Auditory feedback in learning and maintenance of vocal behaviour. Nat Rev Neurosci 1:31-40.

Cheung SW, Nagarajan SS, Schreiner CE, Bedenbaugh PH, Wong A (2005) Plasticity in primary auditory cortex of monkeys with altered vocal production. J Neurosci 25:2490-2503.

Doupe AJ (1997) Song- and order-selective neurons in the songbird anterior forebrain and their emergence during vocal development. J Neurosci 17:1147-1167.

Doupe AJ, Konishi M (1991) Song-selective auditory circuits in the vocal control system of the zebra finch. Proc Natl Acad Sci USA 88:11339-11343.

Doupe AJ, Kuhl PK (1999) Birdsong and human speech: common themes and mechanisms. Annu Rev Neurosci 22:567-631.

Doupe AJ, Perkel DJ, Reiner A, Stern EA (2005) Birdbrains could teach basal ganglia research a new song. Trends Neurosci 28:353-363.

Eales LA (1985) Song learning in zebra finches: some effects of song model availability on what is learnt and when. Animal Behav 33:1293-1300.

Farries MA, Perkel DJ (2000) Electrophysiological properties of avian basal ganglia neurons recorded in vitro. J Neurophysiol 84:2502-2513.

Farries MA, Perkel DJ (2002) A telencephalic nucleus essential for song learning contains neurons with physiological characteristics of both striatum and globus pallidus. J Neurosci 22:3776-3787. 
Goller F, Cooper BG (2004) Peripheral motor dynamics of song production in the zebra finch. Ann NY Acad Sci 1016:130-152.

Goller F, Suthers RA (1996) Role of syringeal muscles in controlling the phonology of bird song. J Neurophysiol 76:287-300.

Green D, Swets J (1966) Signal detection theory and psychophysics. New York: Wiley.

Hessler NA, Doupe AJ (1999) Singing-related neural activity in a dorsal forebrain-basal ganglia circuit of adult zebra finches. J Neurosci 19:10461-10481.

Immelmann K (1969) Song development in the zebra finch and other estrildid finches. In: Bird vocalizations (Hinde RA, ed), pp 61-74. Cambridge, UK: Cambridge UP.

Kao MH, Brainard MS (2006) Lesions of an avian basal ganglia circuit prevent context-dependent changes to song variability. J Neurophysiol 96:1441-1455.

Kao MH, Doupe AJ, Brainard MS (2005) Contributions of an avian basal ganglia-forebrain circuit to real-time modulation of song. Nature 433:638-643.

Konishi M (1965) The role of auditory feedback in the control of vocalization in the white-crowned sparrow. Z Tierpsychol 22:770-783.

Leonardo A (2004) Experimental test of the birdsong error-correction model. Proc Natl Acad Sci USA 101:16935-16940.

Leonardo A, Konishi M (1999) Decrystallization of adult birdsong by perturbation of auditory feedback. Nature 399:466-470.

Linkenhoker BA, von der Ohe CG, Knudsen EI (2005) Anatomical traces of juvenile learning in the auditory system of adult barn owls. Nat Neurosci 8:93-98.

Luo M, Perkel DJ (1999) Long-range GABAergic projection in a circuit essential for vocal learning. J Comp Neurol 403:68-84.

Marler P (1970) A comparative approach to vocal learning: Song development in white-crowned sparrows. J Comp Physiol Psychol 71:1-25.

Marler P, Waser MS (1977) Role of auditory feedback in canary song development. J Comp Physiol Psychol 91:8-16.

Marsden CD, Rothwell JC (1987) The physiology of idiopathic dystonia. Can J Neurol Sci 14:521-527.

Merzenich MM, Kaas JH, Wall J, Nelson RJ, Sur M, Felleman D (1983) Topographic reorganization of somatosensory cortical areas $3 \mathrm{~b}$ and 1 in adult monkeys following restricted deafferentation. Neuroscience 8:33-55.

Merzenich MM, Nelson RJ, Stryker MP, Cynader MS, Schoppmann A, Zook JM (1984) Somatosensory cortical map changes following digit amputation in adult monkeys. J Comp Neurol 224:591-605.

Nordeen KW, Nordeen EJ (1992) Auditory feedback is necessary for the maintenance of stereotyped song in adult zebra finches. Behav Neural Biol 57:58-66.

Nottebohm F, Stokes TM, Leonard CM (1976) Central control of song in the canary, Serinus canarius. J Comp Neurol 165:457-486.

Olveczky BP, Andalman AS, Fee MS (2005) Vocal experimentation in the juvenile songbird requires a basal ganglia circuit. PLoS Biol 3:e153.

Price P (1979) Developmental determinants of structure in zebra finch song. journal of comparative and physiological psychology 93:260-277.
Rosen MJ, Mooney R (2000) Intrinsic and extrinsic contributions to auditory selectivity in a song nucleus critical for vocal plasticity. J Neurosci 20:5437-5448.

Schoenfeld MA, Tempelmann C, Gaul C, Kuhnel GR, Duzel E, Hopf JM, Feistner H, Zierz S, Heinze HJ, Vielhaber S (2005) Functional motor compensation in amyotrophic lateral sclerosis. J Neurol 252:944-952.

Solis MM, Doupe AJ (1997) Anterior forebrain neurons develop selectivity by an intermediate stage of birdsong learning. J Neurosci 17:6447-6462.

Solis MM, Doupe AJ (1999) Contributions of tutor and bird's own song experience to neural selectivity in the songbird anterior forebrain. J Neurosci 19:4559-4584.

Solis MM, Doupe AJ (2000) Compromised neural selectivity for song in birds with impaired sensorimotor learning. Neuron 25:109-121.

Suthers RA, Goller F, Pytte C (1999) The neuromuscular control of birdsong. Philos Trans R Soc Lond B Biol Sci 354:927-939.

Tchernichovski O, Nottebohm F, Ho CE, Pesaran B, Mitra PP (2000) A procedure for an automated measurement of song similarity. Anim Behav 59:1167-1176.

Tessitore A, Esposito F, Monsurro MR, Graziano S, Panza D, Russo A, Migliaccio R, Conforti FL, Morrone R, Quattrone A, Di Salle F, Tedeschi G (2006) Subcortical motor plasticity in patients with sporadic ALS: an fMRI study. Brain Res Bull 69:489-494.

Theunissen FE, Doupe AJ (1998) Temporal and spectral sensitivity of complex auditory neurons in the nucleus $\mathrm{HVc}$ of male zebra finches. J Neurosci 18:3786-3802.

Troyer TW, Doupe AJ (2000) An associational model of birdsong sensorimotor learning I. Efference copy and the learning of song syllables. J Neurophysiol 84:1204-1223.

Wild JM (1993a) The avian nucleus retroambigualis: a nucleus for breathing, singing and calling. Brain Res 606:319-324.

Wild JM (1993b) Descending projections of the songbird nucleus robustus archistriatalis. J Comp Neurol 338:225-241.

Wild JM (1997) Neural pathways for the control of birdsong production. J Neurobiol 33:653-670.

Wild JM, Goller F, Suthers RA (1998) Inspiratory muscle activity during bird song. J Neurobiol 36:441-453.

Williams H, McKibben JR (1992) Changes in stereotyped central motor patterns controlling vocalization are induced by peripheral nerve injury. Behav Neural Biol 57:67-78.

Williams H, Mehta N (1999) Changes in adult zebra finch song require a forebrain nucleus that is not necessary for song production. J Neurobiol 39:14-28.

Williams H, Connor DM, Hill JW (2003) Testosterone decreases the potential for song plasticity in adult male zebra finches. Horm Behav 44:402-412.

Zevin JD, Seidenberg MS, Bottjer SW (2004) Limits on reacquisition of song in adult zebra finches exposed to white noise. J Neurosci 24:5849-5862.

Zheng W, Knudsen EI (1999) Functional selection of adaptive auditory space map by GABAA-mediated inhibition. Science 284:962-965. 